\title{
Respiratory Rhythms of the Predictive Mind
}

\author{
Micah Allen 1,2 , Somogy Varga, Detlef H. Heck4
}

${ }_{1}$ Center of Functionally Integrative Neuroscience, Aarhus University, Denmark

${ }_{2}$ Cambridge Psychiatry, University of Cambridge, United Kingdom

${ }_{3}$ School of Culture and Society, Aarhus University, Denmark

${ }_{4}$ Department of Anatomy and Neurobiology, University of Tennessee

Corresponding author: micah@,cfin.au.dk

Website: https://www.the-ecg.org/

Twitter:@micahgallen

\section{Acknowledgements}

MA is supported by a Lundbeckfonden Fellowship (under Grant [R272-2017-4345]), and a grant from the European Research Council (ERC-StG-948788). The authors thank Francesca Fardo for feedback on the manuscript and figures. Figures 1 and 3 were created using biorender (biorender.com).

\section{Please Cite As:}

Allen, M., Varga, S., \& Heck, D. H. (2022). Respiratory rhythms of the predictive mind. Psychological Review, https://doi.org/10.1037/rev0000391 


\section{ALLEN ET AL., RESPIRATORY RHYTHMS}

\section{Abstract}

Respiratory rhythms sustain biological life, governing the homeostatic exchange of oxygen and carbon dioxide. Until recently however, the influence of breathing on the brain has largely been overlooked. Yet new evidence demonstrates that the act of breathing exerts a substantive, rhythmic influence on perception, emotion, and cognition, largely through the direct modulation of neural oscillations. Here we synthesize these findings to motivate a new predictive coding model of respiratory brain coupling, in which breathing rhythmically modulates both local and global neural gain, to optimize cognitive and affective processing. Our model further explains how respiratory rhythms interact with the topology of the functional connectome, and we highlight key implications for the computational psychiatry of disordered respiratory and interoceptive inference.

Keywords: Respiration, Predictive Coding, Interoception, Neural Gain, Computational Psychiatry 
ALLEN ET AL., RESPIRATORY RHYTHMS

\section{Breathing on the Brain}

Respiration is the physiological process by which living organisms regulate gas exchange in response to environmental demands. This homeostatic process depends on various components, and in mammals, respiration may refer to the part of the process concerned with oxygen being exchanged in the lungs for carbon dioxide from the blood (i.e., external respiration or breathing) or to the part concerned with the passage of oxygen from the blood to the tissues, and the passage of carbon dioxide from the tissues to the blood (i.e., internal respiration). In the context of this article, respiration refers to rhythmic breathing movements, that is, external respiration, which encompasses sensory and motor processes related to breathing.

Control of the respiratory cycle in mammals is primarily achieved by the dorsal and ventral respiratory groups of the brainstem and by top-down modulatory inputs from higher cortical areas such as the insula and cingulate cortex (Del Negro et al., 2018; Herrero et al., 2017). While the link between neuronal processing of olfactory sensory information and the respiratory rhythm has been investigated in some detail (Kay et al., 2009; Kepecs et al., 2006), until recently, the potential for respiratory rhythms to influence other domains of neural processing has received less interest. Recent exceptions include a seminal study focusing on the mouse somatosensory barrel cortex, which showed that the influence of respiration on rhythmic brain activity, including the modulation of power of gamma oscillations, extends to non-olfactory brain areas (Ito et al., 2014). Other recent human and rodent neuroimaging studies report evidence of respiratory phase-amplitude or cross-frequency coupling in the brain, most commonly showing that the power of gamma oscillations modulates in phase with theta oscillations (Canolty \& Knight, 2010; Lisman \& Jensen, 2013). Such theta-gamma phaseamplitude coupling has been linked to cognitive functions such as memory formation in 
ALLEN ET AL., RESPIRATORY RHYTHMS

humans (Lega et al., 2016). In rodents, it has been linked to cognitive performance as measured by a maze task (Tort et al., 2008) or by an item-context association task (Tort et al., 2009).

Given that gamma power in different brain areas has been linked to numerous cognitive functions (e.g., attention, working memory, perceptual grouping), the modulation of gamma power by respiration suggests a direct influence of respiration on cognitive functions. It is important to note that the modulation of gamma oscillation power in non-olfactory brain areas does not require direct projections from the olfactory system but rather propagates indirectly most likely via the dense cortico-cortical and cortico-thalamic networks (Ito et al., 2014). Now, a rapidly growing number of studies demonstrate that respiration not only influences rhythmic brain activity but also modulates behavior across sensory, affective, and cognitive domains (Arshamian et al., 2018; Huijbers et al., 2014; Ito et al., 2014; Kluger et al., 2021b; Kluger \& Gross, 2020a, 2020b; Liu et al., 2017; Nakamura et al., 2018; Perl et al., 2019; Zelano et al., 2016). For recent reviews see: Heck et al., (2017, 2019); Tort et al., (2018).

\section{The sensory domain}

In the sensory domain, recent findings link a variety of perceptual modalities to the respiratory cycle. Perl and colleagues (2019) reported that human participants align the onset of nasal respiration to trial onsets in visuospatial, mathematical, and verbal reasoning tasks. This alignment further bolstered behavior specifically in the visuospatial task, with increases and decreases in accuracy locked to inspiration and expiration-aligned trials, respectively. This behavioral effect was further correlated with localized decreases in alpha and beta band oscillatory rhythms, suggesting a link between changes in neural rhythms and respiratory cycle effects on perception. Another recent study by Kluger and colleagues examined how the respiratory cycle influences visual thresholds, using an adaptive psychophysical task together with MEG recordings (Kluger et al., 2021b). By re-estimating the psychophysical function at 
each bin of the respiratory cycle, the authors found that visual detection became more sensitive during inspiration, and that alpha power was increased during this same time window, suggesting that respiration may help to align rhythms of perception with ongoing neuronal activity. While these studies point towards a potentially unifying role for the modulation of alpha band oscillatory activity by respiration in the visual domain, a recent registered report found no effect of the respiratory cycle on either visual contrast or fearful face discrimination (Mizuhara \& Nittono, 2022). The authors interpreted this null finding as possibly being related to the motor component of their task in which participant actively adjusted stimulus presentation timing. Further, these and other studies have utilized a mixture of different respiratory preprocessing and phase extraction techniques, making it difficult to interpret the heterogeneity of reported findings. Future work could therefore benefit from utilizing registered reports, to better standardize the associated recording and analytic techniques.

In addition to these studies focusing on exteroception, recent work has investigated the influence of respiratory cycles on tactile, interoceptive, and self-related processing. With respect to tactile processing, Grund and colleagues (2022) found that both cardiac and respiratory cycles modulated stimulus sensitivity, with hit rates being maximal at peak expiration and minimal at peak inspiration. With respect to interoception (i.e., the perception of internal, visceral sensations), Zaccaro and colleagues (2022) investigated respiratory cycle effects on cardiac interoception, and reported that heartbeat-evoked cortical potentials were maximal during exhalation versus inhalation. This was further coupled with higher heartbeat tapping accuracy, suggesting that interoceptive sensitivity may be greatest when diaphragmatic pressure increases in the chest during the respiratory baroreflex (Molle \& Coste, 2022; Zaccaro et al., 2022). Finally, with respect to self-related processing, a study reported the existence of an "embreathment illusion" such that projecting respiratory rhythms onto a virtual avatar increased feelings of bodily awareness and immersion (Monti et al., 
ALLEN ET AL., RESPIRATORY RHYTHMS

2019). Other recent studies report that participants were better able to discriminate their own versus others voices during inspiration versus expiration (Orepic et al., 2022), more likely to initiate voluntary actions during inspiration, and that inspiratory phase further enhanced the EEG readiness potential (Park et al., 2020). While it is still too early to elucidate a common effect across these studies, collectively they suggest a potentially important role for respiratory cycles in modulating the somatic, cognitive, and interoceptive components of selfrelated processing (see Azzalini et al., 2019 for review).

\section{The affective domain}

Given the putative link between bodily sensitivity and emotional processing, these findings may further illuminate reports linking the respiratory cycle to affect and emotion (Allen et al., 2019; Apps \& Tsakiris, 2014; Ashhad et al., 2022; Hesp et al., 2021; Nikolova et al., 2021; Seth \& Tsakiris, 2018). A seminal finding in this area was the reported link between intracranial EEG, inspiration, and emotion by Zelano and colleagues (2016). Further highlighting the close linkage between respiratory physiology and emotion more generally, studies find that different positive and negative emotional tasks such as movie watching or stress induction elicit unique profiles of breathing (Bloch et al., 1991; Boiten, 1998; Butler et al., 2006; see Homma \& Masaoka, 2008 for review). A complementary pair of rodent studies investigated the role of respiration in modulating prefrontal cortical rhythms and fear behavior (Bagur et al., 2021; Moberly et al., 2018). In the first, Moberly and colleagues reported that the natural $4 \mathrm{~Hz}$ rhythm of breathing in mice elicited clear oscillations at this frequency in the prelimbic prefrontal cortex, an important region for fear behavior. In a second study, Bagur and colleagues found that the olfactory bulb played a critical role in transmitting this $4 \mathrm{~Hz}$ rhythm to the dorsomedial prefrontal cortex, and that this entrainment effect is specifically involved in regulating freezing behavior during fear conditioning. 


\section{The cognitive domain}

Several studies have investigated how memory and cognition are related to the respiratory cycle. Zelano and colleagues (2016) originally reported that both emotional memory and responses were altered during inspiration versus expiration. A similar study investigated olfactory memory and found that nasal respiration during the encoding phase significantly improved consolidation compared to oral breathing (Arshamian et al., 2018), highlighting again the potentially important role of the olfactory bulb in mediating these effects. Using a delayed-match-to-sample visual recognition task, Nakamura and colleagues (2018) found that locking stimuli to the respiratory phase increased reaction times and decreased accuracy, whereas stimuli that were locked to phase transitions (i.e., stimuli arriving during the transit from inspiration to expiration or vice versa) where not. In what may be the most comprehensive study to date, Johannknecht and Kayser (2022) investigated respiratory cycle effects across six different task paradigms spanning emotion discrimination, visual memory, sound detection, pitch discrimination, and visual motion discrimination. They showed that across all modalities, participants exhibited a tendency to align their breathing rhythm to the timing of the task, inhaling during stimulus presentation and exhaling when responding. Further, in the pitch discrimination, emotion discrimination, and sound detection tasks reaction time was specifically and substantially coupled to the respiratory cycle, exhibiting effect sizes in the 17-61ms range, on par with RT responses to neurostimulation. Given the lack of effects on decision accuracy, these results in particular suggest that respiratory phase may be altering the speed-accuracy trade-off, potentially by increasing neural gain or related evidence accumulation mechanisms (Cheadle et al., 2014; Servan-Schreiber et al., 1990). We will return to the computational and functional implications of this hypothesis in the following sections. 
Three pathways

Collectively, these studies find that respiratory rhythms influence perception, affect, and cognition in a variety of ways. While it is difficult at present to highlight a single thread through these studies, a few clear trends emerge. The first is that, generally speaking, perceptual performance seems to be better at inspiration versus expiration, although this effect likely depends upon the exact task and response structure. Neurophysiologically, these studies highlight respiratory-modulated, task-specific, global changes in neuronal oscillations across all major brain frequencies, with a dominant role for hippocampal theta and sharp wave ripples, prefrontal alpha, and high-frequency gamma oscillations in primary sensory cortices (Ito et al., 2014; Kluger \& Gross, 2020a; Liu et al., 2017). These effects go beyond the simple homeostatic circuits associated with respiratory control and depend upon convergent olfactory, somatosensory, and interoceptive pathways (Figure 1).

A first and particularly important pathway depends upon the mechanical stimulation of the olfactory bulb during intranasal breathing. This pathway plays a major role in regulating the link between respiratory rhythms and the brain. Relative to other sensory domains, olfaction is a phylogenetically ancient neural system, partially bypassing subcortical structures to directly innervate motivational and affective processing hubs such as the orbital frontal cortex (OFC) and amygdala (Insausti et al., 2002; Nigri et al., 2013; Potter \& Nauta, 1979). Ito and colleagues (2014) found that removing the olfactory bulb in mice reduced respiratory-modulated brain oscillations (RMBOs) by as much as $80 \%$. Another recent mouse study utilized optogenetics together with bulbectomy to show that the olfactory bulb transmits respiratory rhythms directly to the dorsomedial prefrontal cortex, enabling respiratory control of fear-related freezing behavior (Bagur et al., 2021). In parallel to these 
ALLEN ET AL., RESPIRATORY RHYTHMS

animal models, human studies have reported unique neural and behavioral effects of intranasal vs intraoral respiration (Perl et al., 2019; Zelano et al., 2016). For example, Zelano and colleagues utilized intracranial recordings to show that intranasal breathing specifically modulates hippocampal theta rhythms as well as performance on memory and emotion tasks (Zelano et al., 2016). On the basis of these findings, we suggest that intranasal respiration plays a dominant role in modulating slower neural oscillations associated with top-down computations, and in particular those that regulate affective and motivational processing.

A second important pathway is the rhythmic progression of somatosensory inputs arising from the diaphragm and chest wall, as respiratory ventilation expands and contracts the lungs at each breath. These regular somatosensory rhythms likely explain recent findings demonstrating RMBOs in the gamma and beta frequency arising from somatosensory cortex and the cerebellum (Kluger \& Gross, 2020a, 2020b). In general, the amplitude and frequency of respiration is closely linked to the overall stability and rhythms of the body - the head, chest, and overall bodily posture oscillate with each breath, an effect which is well-known to both professional marksmen and fMRI specialists seeking to correct motion artefacts in their recordings (Glover et al., 2000). We hypothesize that respiratory-modulated connectivity between the somatomotor cortices and cerebellum helps to align and optimize exteroceptive sensory-motor rhythms (e.g., in the beta range) with the oscillatory activity of the breath.

A third, and perhaps most intriguing pathway, is linked to the role of respiratory rhythms in regulating interoceptive sensations, physiological arousal, and noradrenergic signaling. Interoception is the sensation, perception, and control of the homeostatic condition of the visceral body, and in the respiratory domain, it includes sensations such as air hunger, respiratory frequency, and respiratory effort (Craig, 2002; Garfinkel et al., 2016; Legrand et al., 2021; Nikolova et al., 2022). These sensations, as well as descending respiratory control signals, are carried between the brainstem respiratory nuclei and lungs via two key pathways, 
ALLEN ET AL., RESPIRATORY RHYTHMS

the vagus and phrenic nerves (Bozler \& Burch, 1951). The ascending vagus nerve carries the majority of visceral sensations from the internal organs to the brainstem, with terminals in the respiratory and cardiac medulla as well as the locus coeruleus (LC). In complement, the phrenic nerve originates in the respiratory medulla and carries descending motor signals to the lungs and diaphragm.

Physiological arousal, a key modulator of interoception and emotion, in turn is primarily controlled via the action of noradrenergic neurotransmission as regulated by the LC (Aston-Jones \& Cohen, 2005; Mather et al., 2016). The LC is the primary source of noradrenergic transmission in the mammalian brain, with global, diffuse projections to subcortical and cortical regions which regulate arousal, attention and stress responses (AstonJones \& Cohen, 2005; Benarroch, 2018). An intriguing possibility then is that respiratory rhythms modulate the brain in part by controlling noradrenaline release, creating a causal loop between interoceptive sensation, respiration, and arousal. Based on recent findings by Yackle and colleagues (2017), this influence is likely to occur during each breath cycle and is thus sensitive to changes in respiratory patterns on a breath-by-breath basis. In their study, Yackle and colleagues (2017) identified a subpopulation of neurons in the pre-Botzinger Complex, the primary respiratory rhythm generator of the brainstem, with direct projections to noradrenaline expressing LC neurons. Ablating these connections further eliminated the breath-by-breath control of noradrenaline release, causing mice to exhibit altered arousal responses to exteroceptive stimuli.

Collectively, the emerging consensus view from both animal and human neuroimaging studies places the breath, and its role in modulating neuronal rhythms, as a central regulator of higher-order cognition. Through olfactory, somatosensory, and interoceptive pathways, respiration can be seen as a global rhythm that regulates how and when we process stimuli arising in the body and the world (Varga \& Heck, 2017). In the 
ALLEN ET AL., RESPIRATORY RHYTHMS

body, respiration modulates stability, dynamics, and homeostasis. In the brain, the timing and amplitude of respiration appear to exert diverse influences on neural oscillations, functional connectivity, and behavior. On this basis, we propose that respiration may play a particularly important role in aligning the rhythms of the brain with those of the body and the exteroceptive world.

Our proposal raises a number of intriguing questions, such as: what computational mechanisms mediate these effects? How could we learn from respiration to improve our understanding of the mind and its disorder? To better answer these questions, we now outline a novel predictive coding model of respiratory interoception and breath-brain coupling.

\section{Neural Gain, Respiration, and Precision}

The link between respiration and neural gain is a key element within the theoretical framework we describe here. Neural gain denotes the mathematical function describing the balance between the input to a neuron or population of neurons versus the consequent probability of those neurons firing (Servan-Schreiber et al., 1990). Neurobiologically, gain is controlled by the balance of excitation versus inhibition, such that high excitation enhances the responsiveness of signal-relevant neurons and simultaneously suppresses noise correlations, increasing signal-to-noise ratio (SNR) (Aston-Jones \& Cohen, 2005; Ferguson \& Cardin, 2020). As high gain also triggers stimulus or goal-encoding neurons to fire more coherently, gain directly controls neural oscillations and tuning: for example, high gain in the visual cortex, induced by noradrenergic stimulation, increases both gamma power and SNR of stimulus-detecting neurons (Vinck et al., 2015). At a more global level, adaptive gain control by noradrenergic signaling modulates the global dynamics and topology of the brain's connectome, such that high gain is associated with more stable, less chaotic iterations 
ALLEN ET AL., RESPIRATORY RHYTHMS

between different network topologies, resulting in less variable behavior, increased learning, and more efficient metacognitive control (Eldar et al., 2013, 2016; Shine et al., 2018).

Computationally, local and global neural gain are associated with precision, which is the inverse uncertainty or confidence of prediction and prediction error signals $(H$. R. Brown \& Friston, 2012; Fardo et al., 2017; Warren et al., 2016). As salient sensory inputs induce more robust afferent excitation, feed-forward changes in the gain of deep pyramidal cells increases the precision of ascending prediction errors to induce stronger belief updates (Feldman \& Friston, 2010). Similarly, catecholamines such as dopamine and noradrenaline modulate expected precision by increasing the gain of top-down expectations encoding the long-term volatility of lower-order prediction errors (Hauser et al., 2016; Moran et al., 2013a; Warren et al., 2016). The resulting comparison between feed-forward sensory and top-down expected precision results in second-order precision prediction errors (Balsdon et al., 2020; Guggenmos et al., 2016), whose estimation and minimization dynamically reshapes patterns of global neural connectivity.

Our model predicts that respiration shapes neural gain and precision at multiple hierarchical levels. As the body moves in rhythm with the breath, slow-oscillating changes in the breath also modulate the variability of sensory inputs and motor responses. Respiratorylocked changes in arterial pulsation, blood oxygenation, and blood temperature also mechanically alter baseline firing rates and neural noise correlations, further linking the gain of local neural populations to respiratory dynamics (Chow et al., 2020; Karbowski, 2009). One recent computational model captured these dynamics by linking periodic sensory attenuation to cardiac and respiratory cycles, demonstrating that even small, but consistent changes in visceral rhythms exert substantive influences on in silico behavior and exteroceptive precision (Allen et al., 2019). Respiratory rhythms also directly elicit noradrenaline release in the locus coeruleus, suggesting a close linkage of global gain and 
ALLEN ET AL., RESPIRATORY RHYTHMS

breathing patterns (Yackle et al., 2017). Altogether, our account suggests that the heterogenous control of gain by respiratory rhythms helps to explain their observed influence on brain oscillations, behavior, and neural computation.

\section{The Predictive Processing Framework}

The cognitive sciences have recently seen a resurgence of interest in predictive processing theories of brain function (Bastos et al., 2012; Clark, 2013; K. Friston, 2018; K. Friston \& Kiebel, 2009; Hohwy, 2013). These approaches invert the classical stimulus-response view of the mind, in which computation primarily proceeds in a bottom-up fashion, to recast neural processing as minimizing the mismatch between top-down predictions and bottom-up prediction errors. Here the hierarchical networks of the brain embody a probabilistic generative model which encodes the likelihood of sensory inputs given prior beliefs regarding their hidden causes. These hidden causes can include the statistical regularities of the environment, and the body itself in terms of the sensory organs and effectors that couple the agent to its environment.

In the nervous system, predictive processing is thought to be achieved by means of hierarchical predictive coding, an algorithm for computing the mismatch (prediction error) between probabilistic prior beliefs and ongoing sensory inputs (Adams et al., 2015; K. Friston \& Kiebel, 2009; Rao \& Ballard, 1999). On this model, top-down connections encode a prior probability (i.e. prediction) over some hidden sensory input, whereas feed-forward connections communicate sensory prediction errors. The balance between these signals is regulated by their precision, or inverse noise, which is implemented in neural circuits by the control of post-synaptic gain (Pinotsis et al., 2019).

The interaction between top-down priors and bottom-up prediction errors is further regulated by the joint precision of these signals, with the more precise signal governing belief 
ALLEN ET AL., RESPIRATORY RHYTHMS

updates. This means that, for example, if priors are inherently more precise than incoming sensory inputs, then perception is expected to be prior dominated, more akin to a hallucination than a veridical percept (Corlett et al., 2019). Conversely, very precise sensory inputs associated with particularly strong or salient sensations can be expected to override even highly precise priors. The global optimization of precision is therefore cast as a mechanism governing attentional selection, salience, and metacognition (Allen et al., 2016; Fardo et al., 2017; Feldman \& Friston, 2010; Kanai et al., 2015).

Neurobiologically, mounting evidence suggests that these computations are carried out by a hierarchical canonical microcircuit motif in which the superficial cortical layers convey predictions to the pyramidal cells of deep cortical layers, which in turn project prediction errors to the superficial layers at the next level of cortical processing (Adams et al., 2015; Bastos et al., 2012). Within this microcircuit motif, precision is controlled by neural gain, i.e. the balance of local excitation versus inhibition within each neural population (Moran et al., 2013b; Warren et al., 2016).

At each level of the hierarchy, these distinct prediction, prediction error, and associated precision signals are nested together to encode the complex web of causal relations embedding the perceiving, acting subject into its bodily milieu and external environment. In this way, the minimization of prediction error proceeds from the most basic sensory-motor levels of the brain to progressively more integrative, multi-modal representations of the self (Allen \& Friston, 2016; Apps \& Tsakiris, 2014).

Along the spatio-temporal dimension of the cortical hierarchy, one can also distinguish between low-level, predictive coding based "first order" streams which encode more immediate, categorial outcomes (e.g., perceiving an angry or happy face) versus "second order" prediction streams which encode precision prediction errors (e.g., how confident I am that my perception of a happy face is correct), or the expected precision, of the 
ALLEN ET AL., RESPIRATORY RHYTHMS

first order outcomes (K. Friston, 2018; K. J. Friston et al., 2018; Nikolova et al., 2021). The second order stream is engaged in 'deep' temporal inference over continuous outcomes, linking the immediate moment-to-moment prediction of sensory inputs to longer term contingencies between the agent and the world (Parr \& Friston, 2017). Second-order streams are typically understood as performing a metacognitive function, such as the optimization of attentional control, the assessment of the fidelity or accuracy of first order percepts, or the learning of internal statistics related to lower-order sensorimotor processes (Feldman \& Friston, 2010; Hesp et al., 2021; Nikolova et al., 2021; Whyte \& Smith, 2021). These streams also map onto a temporal hierarchy of neural oscillations, in which lower-order prediction streams map onto faster neural rhythms (e.g., gamma, beta) and higher-order streams onto slower rhythms, (e.g., alpha, theta) (Bastos et al., 2020; Pinotsis et al., 2019).

Here, we build on recent extensions of this approach to interoceptive perception (Allen, 2020; Barrett \& Simmons, 2015; Petzschner et al., 2021; Seth, 2013; Seth \& Tsakiris, 2018) to propose a new predictive coding model of respiratory interoception and breath-brain coupling, illustrated in Figure 2. Our model attempts to unite the disparate findings and neural pathways reviewed in the previous section, suggesting that respiration modulates cognition, perception, and affect by altering neural gain, and that this in turn explains the global effect of respiratory rhythms on neural synchrony and the functional connectome.

\section{A Predictive Coding Model of Respiratory Interoception}

Our model ascribes automatic, unconscious respiratory control to first order interoceptive prediction error streams circumscribed within the brainstem, while conscious interoceptive perception and metacognitive respiratory control correspond to hierarchically deeper second order streams, situated above the brainstem in the insular, cingulate, and prefrontal cortices. This mapping then predicts unique targets for respiratory modulation across the cortical 
ALLEN ET AL., RESPIRATORY RHYTHMS

hierarchy, considering the three neuroanatomical pathways described earlier. On this account, brainstem nuclei of the respiratory nuclei monitor the dynamics of ascending physiological signals via vagal inputs, which encode the breath-by-breath dynamics of respiratory frequency, breathing effort, blood CO2 levels, and other homeostatic parameters. Here, simple prospective control loops, similar to those found in the motor domain (Körding \& Wolpert, 2004; Wolpert, 1997), maintain these parameters according to innate homeostatic set-points by minimizing the difference between afferent visceromotor predictions and the reafferent signaling of resulting changes in respiratory dynamics modulated via the descending phrenic nerves. In this sense, the respiratory nuclei together with basic physiological reflex arcs form a simple respiratory comparator, maintaining the physiological parameters that are necessary for life with little need for higher-order inputs or conscious control.

Beyond mere homeostatic control, this process of comparison generates progressively higher-order prediction errors as one moves upwards in the respiratory hierarchy, from basic brainstem medulla, on to subcortical and cortical regions associated with the conscious perception and metacognitive control of interoception, such as the insula, cingulate, and ventromedial prefrontal cortices (Craig, 2002; Evans et al., 2002; McKay et al., 2003; Petzschner et al., 2021). Along this ascending pathway, respiratory prediction errors are progressively more removed from immediate sensory inputs, encoding deviance from the expected precision of respiration at longer timescales. We therefore associate conscious respiratory interoception and control with the minimization of these second-order precisionprediction errors (Ainley et al., 2016), which encode the predictability of lower-order respiratory prediction rhythms.

Interoception has been previously described in terms of multiple hierarchical levels, corresponding to basic ascending viscero-sensory inputs, conscious interoceptive perception or 'interoceptive accuracy', and interoceptive metacognition or 'interoceptive awareness' 
ALLEN ET AL., RESPIRATORY RHYTHMS

(Garfinkel et al., 2015; Petzschner et al., 2021; Smith et al., 2017). Our proposed predictive coding hierarchy, illustrated in Figure 3, recasts these functional levels onto the previously described gradient of interoceptive and allostatic prediction errors, with the lowest levels of viscero-sensory and viscero-motor loops associated with unconscious homeostatic control mapping onto low-level comparators, more mid-level, conscious perception of the breath to intermediate or "association" level predictive loops, and higher-order metacognitive learning, attentional control, and awareness associated with the highest level of this hierarchy. As such, we position automatic homeostatic control of respiration as a process involving comparative predictive coding loops, conscious perception as a cortical generative model situated one level above these loops (i.e., integrating interoceptive, tactile, and exteroceptive PEs into a perceptual model), and interoceptive metacognition with computationally 'deep' temporal inference over the expected precision of these lower-order sensory streams.

A unique feature of our model is that it situates higher-order respiratory-brain interaction as being governed by a process of deep temporal inference, which links the firstorder respiratory outcomes to more general, self-related processes in a form of allostatic inference (Hesp et al., 2021). Allostatic inference refers to the prospective control of homeostasis through interaction with the environment, as opposed to interoceptive inference, which merely maintains homeostasis via predictive coding. As the second-order stream is inherently concerned with the long-term predictability (i.e., expected precision) of respiration, this stream must incorporate dyadic relationships between the agent and its environment with respiratory contingencies. For example, if an agent expects to encounter a threatening environment or interaction, then it can also predict an increase in the precision of lower-order respiratory prediction errors. In this sense, second-order respiratory inference intermingles the affective valence of various situations with changes in respiratory rhythms. 
ALLEN ET AL., RESPIRATORY RHYTHMS

We propose then that we most likely become aware of and exert conscious control over our respiration whenever higher-order, allostatic respiratory predictions unexpectedly change, i.e., when the predictability of respiration in relation to salient events in the world shifts unexpectedly. On our model, conscious respiratory awareness and control are primarily concerned with the expected precision of respiratory rhythms, rather than the low-level viscero-sensory prediction errors per se.

\section{Breath-brain coupling and global neuronal gain}

While our predictive coding model of respiratory interoception explains within-domain processing and control of respiratory sensations at successive levels of the interoceptive and allostatic hierarchy, what of the more global, cross-domain and oscillatory effects ascribed to breath-brain coupling? We argue that these depend upon the interplay of respiratory rhythms and global neuronal gain. Starting from the low-level brainstem circuits, evidence from Yackle and colleagues suggests that breath-by-breath changes in respiration are directly linked to noradrenaline release in the LC (Yackle et al., 2017). The LC in turn plays a wellestablished role in regulating gain globally across the cortical and subcortical hierarchy, with noradrenergic terminals modulating the excitability of wide-ranging circuits including prefrontal and cingulate areas associated with cognitive control, right down to low-level populations of neurons in the primary visual cortex (Ferguson \& Cardin, 2020; Grueschow et al., 2020; Vinck et al., 2015). Respiratory rhythms thus proffer a unique means by which the physiological rhythms of the body can potentially alter neural gain across the cortex.

This hypothesis has several implications for understanding breath-brain coupling and respiratory predictive processing. Numerous studies now suggest that much like the trained marksmen who couple their pull of the trigger to the onset of inspiration (Konttinen \& Lyytinen, 1992), cognitive, affective, and motor behavior in a variety of domains depends in 
ALLEN ET AL., RESPIRATORY RHYTHMS

part upon the timing and amplitude of the respiratory cycle (Arshamian et al., 2018;

Nakamura et al., 2018; Zelano et al., 2016). However, these effects are largely heterogenous, depending on a variety of different neural rhythms and experimental manipulations. If respiration alters neural gain by means of noradrenergic modulation, then this effect could be explained by the heterogeneity of noradrenaline projections in the brain and through the inherently context-sensitive nature of adaptive gain control.

For example, arousal and cognitive performance are related according to the wellknown Yerkes-Dodson curve in which moderate, but not high or low, arousal is associated with optimal behavioral outcomes (Aston-Jones \& Cohen, 2005). Similarly, the balance of tonic versus phasic noradrenaline response is closely related to wakefulness, as well as the balance between more exploration versus exploitation driven decision-making. This balance has been modeled as an overall index of global neuronal gain, and further linked to alterations in the dynamics and topology of the functional connectome (Eldar et al., 2013; Shine et al., 2018). The impact of respiration on behavior and neural processing can therefore be expected to be highly sensitive to time and context, depending on both baseline arousal and the overall experimental manipulation. Further, whereas breath-to-breath respiratory parameters can be expected to interact primarily with phasic noradrenergic responses modulating immediate responses, longer-term shifts in respiratory behavior could potentially reshape the overall pattern of neural connections by altering overall brain connectivity patterns.

In terms of predictive processing, this control of neural gain by noradrenaline has been causally linked to precision (Warren et al., 2016), and effectively regulates the signal-tonoise ratio of neural populations by enhancing the activity of target-responsive neurons and suppressing local noise correlations. Psychologically, we propose that this global mechanism helps to align local neural oscillations with endogenous, breath-driven fluctuations in neural excitation to improve goal-oriented cognition and perceptual target detection. As such, the 
ALLEN ET AL., RESPIRATORY RHYTHMS

breath-by-breath control of noradrenaline further modulates the precision of prediction and prediction error signals in a non-specific fashion throughout the brain, and proffers a putative mechanistic explanation of effects whereby perceptual sensitivity or memory encoding varies as a function of the respiratory cycle (Arshamian et al., 2018; Nakamura et al., 2018; Zelano et al., 2016). The implication is that at multiple levels of the cortical hierarchy, respiration can modulate either the precision of low-level interoceptive prediction errors, second-order precision-prediction errors, or more global topological characteristics of neural gain. In all three cases, optimal behavior will depend at least in part on aligning respiratory rhythms with ongoing neural and environmental oscillations. We consider this as a case of respiratory active inference - a proposed neuronal mechanism, in which organisms reduce prediction error and/or optimize precision by altering when and how they breath, rather than by updating an internal model of the world (Boyadzhieva \& Kayhan, 2021).

\section{Long-term changes in respiratory physiology and the functional connectome}

As electrophysiology advanced from single-site to multi-site recordings, neuronal activity at two independent sites was often found to correlated, suggesting common input and/or neuronal interactions between the two structures. The concept of functional connectivity was developed as a quantitative description of such observations (Fox \& Raichle, 2007; K. J. Friston, 2011). The functional connectome, i.e. the connectivity fingerprint which defines the global topology of brain networks, is not static but can undergo rapid, context dependent changes that have been linked to the release of neuromodulatory transmitters, such as dopamine and noradrenaline (Shine et al., 2016, 2019; Zerbi et al., 2019). Noradrenaline specifically has been shown to enhance long-range functional network connectivity and task performance in humans (Eldar et al., 2013; Shine et al., 2018). 
ALLEN ET AL., RESPIRATORY RHYTHMS

Via its influence on neuronal excitability and neuronal oscillations, respiration is likely to influence the brain's functional connectome at multiple time-scales. At shorter intervals, respiration modulates neuronal excitability and the power of neuronal oscillations including gamma oscillations (Ito et al., 2014; Kluger et al., 2021a; Varga \& Heck, 2017), both of which have been linked to alterations in synaptic plasticity (Galuske et al., 2019; Rebola et al., 2010), suggesting that breathing can contribute to sustained changes in network topology and neurotransmission. The breath-by-breath modulation of LC activity by respiration presents another route by which respiratory patterns can alter both sustained statebased neural activity and evoked responses to environmental stimuli (Yackle et al., 2017). Noradrenaline directly and rapidly modulates the functional connectome (Zerbi et al., 2019), and it is likely that respiratory-locked changes in synaptic plasticity and neuromodulation interact to shape overall brain connectivity.

We also propose that respiration is also likely to have a long-term modulatory influence on the functional connectome. This long-term influence depends on individual differences in respiratory behavior. Shallow vs. deep breathing, predominantly intranasal or intraoral breathing, variability of the respiratory rhythm, and possible genetic differences in the magnitude of respiratory modulation of brain activity, are all aspects of respiration which, when considered over months and years, will have accumulative effects on the connectivity and function of any brain structure whose activity is modulated by respiration. This influence is likely to start early in life with activity dependent synaptic plasticity playing a crucial role in brain development (Chaudhury et al., 2016). Overall, on the basis of the material considered here, we suspect that systematic, long-term observations of respiratory behavior would provide surprising new insights into the correlation of respiratory rhythms and health, and open avenues for targeted breath-based treatment development for cognitive disorders, such as respiratory active interference. 


\section{Respiratory active inference - breathing at the right time}

Active Inference is a computational process theory derived from the Free Energy Principle, which argues that biological agents minimize prediction error in two ways: either by updating their beliefs, or by acting on the world so as to make sensory inputs better conform to expectations (Adams et al., 2015; K. Friston, 2013, 2018). On our model, respiratory dynamics are closely coupled to neural gain, resulting in more or less precise sensory-motor information at different phases and rhythms of the breath. This is coherent with theories of interoceptive self-inference, which argue that visceral rhythms in general are closely coupled to expected precision (Ainley et al., 2016; Allen, 2020; Allen et al., 2020; Nikolova et al., 2021). However, respiration differs from other interoceptive modalities in one key aspect: it is directly amenable to conscious control and can modulate other interoceptive variables such as cardiac arousal. This raises the prospect of respiratory-brain coupling as a unique domain of respiratory active inference, in which agents prospectively monitor and control their own visceral dynamics - breathing at the right time - to optimize sensory precision when it is needed most. Perhaps the many athletic, contemplative, and martial-arts traditions that emphasize working with the breath for peak performance offer unique insights into this process of respiratory alignment.

This notion of respiratory active inference further highlights the unique evolutionary role of respiration in the brain. The neural mechanisms linked to the control of respiration are arguably among the phylogenetically oldest of all neural functions and support two vital processes, gas exchange (Hsia et al., 2013) and olfactory perception in terrestrial vertebrates (Kay et al., 2009; Kepecs et al., 2006). During the detection of olfactory information (e.g., "sniffing" behavior) the respiratory rhythm is altered from the rhythm determined by blood oxygen homeostasis to a sniffing pattern optimized for olfactory perception (Kay, 2014; 
ALLEN ET AL., RESPIRATORY RHYTHMS

Sobel et al., 1998). Breathing can also be deliberately controlled to generate vocalizations, and - possibly unique to humans - to generate precisely controlled airflow through wind instruments. Given the relative computational complexity of evolving an elaborate multicontrol scheme, it is natural to wonder what benefit this adaption may have had. If, however, agents can learn to optimize their own somato-motor, affective, or precision dynamics through the active modulation of respiration, as we have here argued, then this may provide some insight into why humans evolved this unique ability. Alternatively, it may be a happy accident of the evolution of language that we gained another tool by which to learn about, and ultimately improve our abilities.

\section{Computational Psychiatry of Respiratory Rhythms}

We have described several complementary pathways by which respiratory rhythms interact with the computations of the predictive mind. In particular, we have outlined a predictive coding view of how respiratory control and awareness depends on the hierarchical integration of interoceptive prediction errors in the brainstem, midbrain, insula, and prefrontal cortex. These prediction errors not only control respiratory control and interoception, but as they ascend the cortical hierarchy, become increasingly integrated with emotional and affective processing streams. We further described how the unique coupling of respiration to neural gain and sensory precision renders it a key modality for shaping the functional connectome, and by extension, metacognition, salience, and learning. The unique ability to take conscious control of respiration further raises the possibility that agents optimize their own neural gain by breathing at the right time, coupling their respiratory frequency to the timing of action and sensation to maximize signal-to-noise ratio and align neural synchrony to these events. Here, we consider the implications of this model of the emerging computational psychiatry of respiratory interoception. 
ALLEN ET AL., RESPIRATORY RHYTHMS

Disturbances in primary interoceptive signaling between the brain and body, and in explicit interoceptive awareness, are implicated in a wide variety of psychiatric and neurological disorders (Khalsa et al., 2018; Owens et al., 2018). Respiratory interoception is an especially relevant modality for understanding psychiatric disorders. Feelings of breathlessness, or dyspnea, are major symptoms of anxiety and panic attack, and if they are chronic, they are associated with substantial increases in depression and anxiety diagnoses (Kellner et al., 1992; Manning \& Schwartzstein, 1995).

Accurate sensory perception of respiration is of crucial importance for self-monitoring and recognition of potential health problems, such as asthma or chronic obstructive plulmonary disease (COPD). Giardino et al. (2010) compared the subjective dyspnea ratings (i.e. "how difficult is it to breathe") in response to inspiratory resistive loads of COPD patients who also suffer from panic disorders with patients who do not. COPD patients who suffered from panic disorder reported significantly higher dyspnea than patients in the control group. Results from a study by Leupoldt et al. (2011) suggest that these subjective reports are reflected in the neuronal correlates of perception of respiration in the cerebral cortex. The authors exposed healthy individuals with low and high levels of anxiety to neutral and unpleasant affective visual stimuli and compared their respiratory-related evoked potentials (RREP), a measure of neural activity prompted by short inspiratory occlusions. Their results showed that high-anxiety levels were correlated with higher amplitude P2 and P3 components of the RREP, which are associated with higher order processing of the respiration sensation. While it is difficult to draw clear conclusions about causality here, the general assumption in the literature is that anxiety levels affect the higher-order neural processing of respiratory sensations. The alternative possibility that increased sensitivity to respiratory sensations leads to higher levels of anxiety remain to be explored. 
ALLEN ET AL., RESPIRATORY RHYTHMS

The close linkage between respiratory psychophysiology, affect, and mental health is particularly salient in light of the COVID-19 pandemic, in which more than 150 million people so far (spring of 2022) have been infected with the virus. Of these, nearly 13 million survivors (approximately 15\%) are estimated to suffer from what has become known as "long covid", the persistence of respiratory and other symptoms weeks or even months following infection, even in the absence of any known physiological damage (Sudre et al., 2021). Indeed, recent studies have found that as many as one in three survivors of COVID-19 are diagnosed with a psychiatric illness (most commonly anxiety or depression) within 6 months of infection (Taquet et al., 2021), and these effects are statistically associated with respiratory and other persistent physiological symptoms (Naidu et al., 2021). Although the exact nature of these effects is still unknown, it is likely that the worldwide spikes in depression, anxiety, and other psychiatric diagnoses are at least in part related to the pandemic fundamentally changing the ways in which we relate to our respiratory sensations. It is therefore imperative that we develop targeted, mechanism-based means to intervene on and remediate altered respiratory function to help stem this tide of mental illness.

Luckily, there is substantive evidence that consciously attending to respiratory behavior offers a means of regulating emotional states and cognitive processes (Arch \& Craske, 2006; R. P. Brown et al., 2013; Grossman \& Christensen, 2007). This knowledge has been an integral part of the traditional practices of yogic breathing (R. P. Brown et al., 2013; Jella \& Shannahoff-khalsa, 1993; Stančák \& Kuna, 1994), and forms of controlled slow breathing are also used by military personnel to reduce stress and regain focus in extremely stressful situations (Grossman \& Christensen, 2007). Similarly, long-term breath retraining, which instructs users to rely more on intranasal breathing, can be delivered electronically and is associated with substantive decreases in both anxiety and asthma attacks in sufferers (Arden-Close et al., 2019; Tweeddale et al., 1994). 
Developing a computational approach to modelling the close linkages between mental health symptoms such as anxiety and respiration can help us to better understand the respiratory patterns and behaviors that underly the pathogenesis of mental illness. Indeed, exciting new work on the computational modelling of respiration and mental health has already begun to emerge (Faull et al., 2017; Harrison, Garfinkel, et al., 2021; Harrison, Nanz, et al., 2021). By decomposing the specific computational mechanisms by which respiration entrains neural gain, we may be able to adapt and optimize both acute and chronic breathretraining interventions, resulting in better treatment. Further, in the search for psychiatric biomarkers, quantifying and clustering the unique computational fingerprints of breath-brain interactions that underlie aberrant learning and decision-making in mood and other psychiatric disorders (Pulcu \& Browning, 2019), may ultimately inform our understanding and ability to treat these illnesses.

\section{Empirical Predictions and Open Questions}

In many cases, putative theoretical or computational models of the brain raise important questions about falsifiability. Reflecting on this issue, in this section we briefly outline a few key hypotheses which we believe could readily be tested and ultimately falsified through future empirical and computational work. It is worth noting that in general, computational models are conceived of as abstractions from nature rather than absolute end points, and the ultimate test of any model is its ability to explain the data while achieving maximum parsimony. In this sense then, evaluating the predictions made here is a two-step process, involving both empirical model comparison and the possible falsification of predictions made when running those same models in a forward inference model (Osborne et al., 2014; Palminteri et al., 2017; Wilson \& Collins, 2019). For example, here we (echoing other recent theorists) predict that low-level, homeostatic comparator loops will engage in predictive coding to maintain respiratory variables along specific set-points (Allen, 2020; Barrett \& 
ALLEN ET AL., RESPIRATORY RHYTHMS

Simmons, 2015; Petzschner et al., 2021; Stephan et al., 2016). This prediction can be tested directly, first by recording neuronal activity in these regions during various perturbations of respiratory drive, and second by fitting predictive coding models to the obtained data. Should a formal model comparison then indicate that a simpler, non-predictive model best fit the data, then it would be a strike against the theory. At least one recent paper has already taken a strong step in this direction, using computational model comparison to find the best fitting explanation for how participants learn about respiratory stimuli (Harrison, Köchli, et al., 2021). Similar work is underway in the cardiac and gastric domains (Allen et al., 2019; Smith et al., 2021).

A more important, but harder still step is to go beyond relative model comparison to direct falsification. In this sense, assume that in the previous example, it has been shown that generally speaking, a predictive coding model best fits the respiratory nuclei recordings.

Here, this model should then be simulated so as to make predictions about what will happen to respiratory behavior (i.e., frequency, depth, etc) when the putative prediction error cells are perturbed experimentally (i.e., by lesion, injection, or other causal intervention). This prediction must then be compared by direct causal intervention: if the intervention fails to produce the predicted response, then the model is falsified.

It is with this notion of finding model evidence and falsification in mind that we make the following (speculative) empirical predictions, which we imagine should be productive for future empirical work on respiratory brain-coupling and predictive processing. First, we hypothesize that brainstem circuits will accomplish respiratory control via homeostatic predictive coding. Likewise, we anticipate that higher order respiratory perception and associative learning will be accomplished by multisensory prediction error units in the insular, somatosensory, and cingulate cortices. Finally, we expect that higher-order metacognitive respiratory control - such as the prediction and learning of respiratory 
ALLEN ET AL., RESPIRATORY RHYTHMS

volatility - will be accomplished by ventromedial prefrontal circuits encoding precision prediction errors.

It is worth noting that, a recent high-profile paper from Harrison and colleagues (2021) has actually already provided at least partial evidence in support this hypothesis. Using a straightforward probabilistic respiratory learning task together with computational modelling, Harrison-Faull and colleagues demonstrated that respiratory-exteroceptive learning was best described by a Bayesian reinforcement learning model, and that respiratory prediction errors and their precision were associated with activations of the insular cortex. Expanding this approach to include dynamic changes in the underlying probability transitions (i.e., manipulating expected respiratory volatility itself), we would then expect that ventromedial prefrontal responses would best fit adaptive responses to respiratory volatility. Finally, falsification here could proceed by adapting these paradigms to animal models and using optogenetic and/or other causal interventions (e.g., transcranial ultrasound stimulation) to interfere with these circuits. If the ability to track and learn from respiratory volatility is not abolished, then the theory should be revised.

Finally, we predict that the intra-nasal pathway for respiratory-brain coupling described earlier will play an especially important role in modulating sensitivity to respiratory volatility. What is unique about this pathway is precisely that is offers a means by which respiratory rhythms can largely bypass the underlying cortical hierarchy and more or less directly perturb the relevant prefrontal circuits. We would expect that RMBOs in these brain regions are likely to correlate with individual biases in respiratory volatility tracking, and could also delineate one means by which such biases could lead to chronic mood and/or respiratory disorders - as aberrant tracking of expected uncertainty (volatility) has itself been proffered as a general mechanism underlying disorders of anxiety and depression (Pulcu \& Browning, 2019). 


\section{ALLEN ET AL., RESPIRATORY RHYTHMS}

\section{Concluding Remarks}

The neural mechanisms underlying the influence of breathing on brain activity are rapidly becoming a major focus in the emerging fields of brain-body neuroscience and interoception. This newly emerging domain brings with it unique opportunities for translational, computational, and clinical neuroscience investigations probing the breadth, nature, and extent of breath-brain interactions. In the hopes of providing a meaningful roadmap to discovery, we here outlined some of the major neuroanatomical pathways by which respiratory rhythms modulate brain oscillations and shape behavior. Further, we motivated a novel predictive coding model of the relevant effects, in which respiration acts a global rhythm modulating neural gain and expected precision at multiple levels of the cortical hierarchy. We expect that understanding how and why we engage with respiratory active inference - learning to breath at the right time - will reveal deep linkages between neural computation, mental health, and respiratory function. 
ALLEN ET AL., RESPIRATORY RHYTHMS

References

Adams, R., Friston, K., \& Bastos, A. (2015). Active Inference, Predictive Coding and Cortical Architecture. Recent Advances On The Modular Organization Of The Cortex, 97-121. https://doi.org/10.1007/978-94-017-9900-3_7

Ainley, V., Apps, M. A. J., Fotopoulou, A., \& Tsakiris, M. (2016). 'Bodily precision': A predictive coding account of individual differences in interoceptive accuracy. Philosophical Transactions of the Royal Society B: Biological Sciences, 371(1708), 20160003.

https://doi.org/10.1098/rstb.2016.0003

Allen, M. (2020). Unravelling the Neurobiology of Interoceptive Inference. Trends in Cognitive Sciences, 24(4), 265-266. https://doi.org/10.1016/j.tics.2020.02.002

Allen, M., Frank, D., Schwarzkopf, D. S., Fardo, F., Winston, J. S., Hauser, T. U., \& Rees, G. (2016). Unexpected arousal modulates the influence of sensory noise on confidence. ELife, 5, e18103. https://doi.org/10.7554/eLife.18103

Allen, M., \& Friston, K. J. (2016). From cognitivism to autopoiesis: Towards a computational framework for the embodied mind. Synthese. https://doi.org/10.1007/s11229-016-1288-5

Allen, M., Legrand, N., Correa, C. M. C., \& Fardo, F. (2020). Thinking through prior bodies: Autonomic uncertainty and interoceptive self-inference. Behavioral and Brain Sciences, 43. https://doi.org/10.1017/\$0140525X19002899

Allen, M., Levy, A., Parr, T., \& Friston, K. J. (2019). In the Body's Eye: The Computational Anatomy of Interoceptive Inference. BioRxiv, 603928. https://doi.org/10.1101/603928

Apps, M. A. J., \& Tsakiris, M. (2014). The free-energy self: A predictive coding account of selfrecognition. Neuroscience \& Biobehavioral Reviews, 41, 85-97. https://doi.org/10.1016/j.neubiorev.2013.01.029

Arch, J. J., \& Craske, M. G. (2006). Mechanisms of mindfulness: Emotion regulation following a focused breathing induction. Behaviour Research and Therapy, 44(12), 1849-1858. https://doi.org/10.1016/j.brat.2005.12.007 
ALLEN ET AL., RESPIRATORY RHYTHMS

Arden-Close, E. J., Kirby, S. E., Yardley, L., Bruton, A., Ainsworth, B., \& Thomas, D. M. (2019). Evaluation of a breathing retraining intervention to improve quality of life in asthma: Quantitative process analysis of the BREATHE randomized controlled trial. Clinical Rehabilitation, 33(7), 1139-1149. https://doi.org/10.1177/0269215519832942

Arshamian, A., Iravani, B., Majid, A., \& Lundström, J. N. (2018). Respiration Modulates Olfactory Memory Consolidation in Humans. The Journal of Neuroscience, 38(48), 10286. https://doi.org/10.1523/JNEUROSCI.3360-17.2018

Ashhad, S., Kam, K., Negro, C. A. D., \& Feldman, J. L. (2022). Breathing Rhythm and Pattern and Their Influence on Emotion. Annual Review of Neuroscience, 45(1), null. https://doi.org/10.1146/annurev-neuro-090121-014424

Aston-Jones, G., \& Cohen, J. D. (2005). An integrative theory of locus coeruleus-norepinephrine function: Adaptive gain and optimal performance. Annu. Rev. Neurosci., 28, 403-450.

Azzalini, D., Rebollo, I., \& Tallon-Baudry, C. (2019). Visceral Signals Shape Brain Dynamics and Cognition. Trends in Cognitive Sciences. https://doi.org/10.1016/j.tics.2019.03.007

Bagur, S., Lefort, J. M., Lacroix, M. M., de Lavilléon, G., Herry, C., Chouvaeff, M., Billand, C., Geoffroy, H., \& Benchenane, K. (2021). Breathing-driven prefrontal oscillations regulate maintenance of conditioned-fear evoked freezing independently of initiation. Nature Communications, 12(1), 2605. https://doi.org/10.1038/s41467-021-22798-6

Balsdon, T., Wyart, V., \& Mamassian, P. (2020). Confidence controls perceptual evidence accumulation. Nature Communications, 11(1), 1753. https://doi.org/10.1038/s41467-02015561-w

Barrett, L. F., \& Simmons, W. K. (2015). Interoceptive predictions in the brain. Nature Reviews Neuroscience, 16(7), 419-429. https://doi.org/10.1038/nrn3950

Bastos, A. M., Lundqvist, M., Waite, A. S., Kopell, N., \& Miller, E. K. (2020). Layer and rhythm specificity for predictive routing. Proceedings of the National Academy of Sciences, 117(49), 31459-31469. 
ALLEN ET AL., RESPIRATORY RHYTHMS

Bastos, A. M., Usrey, W. M., Adams, R. A., Mangun, G. R., Fries, P., \& Friston, K. J. (2012). Canonical Microcircuits for Predictive Coding. Neuron, 76(4), 695-711. https://doi.org/10.1016/j.neuron.2012.10.038

Benarroch, E. E. (2018). Locus coeruleus. Cell and Tissue Research, 373(1), 221-232. https://doi.org/10.1007/s00441-017-2649-1

Bloch, S., Lemeignan, M., \& Aguilera-T, N. (1991). Specific respiratory patterns distinguish among human basic emotions. International Journal of Psychophysiology, 11(2), 141-154. https://doi.org/10.1016/0167-8760(91)90006-J

Boiten, F. A. (1998). The effects of emotional behaviour on components of the respiratory cycle. Biological Psychology, 49(1), 29-51. https://doi.org/10.1016/S0301-0511(98)00025-8

Boyadzhieva, A., \& Kayhan, E. (2021). Keeping the Breath in Mind: Respiration, Neural Oscillations, and the Free Energy Principle. Frontiers in Neuroscience, 15. https://www.frontiersin.org/article/10.3389/fnins.2021.647579

Bozler, E., \& Burch, B. H. (1951). Role of the Vagus in the Control of Respiration. American Journal of Physiology-Legacy Content, 166(2), 255-261. https://doi.org/10.1152/ajplegacy.1951.166.2.255

Brown, H. R., \& Friston, K. J. (2012). Dynamic causal modelling of precision and synaptic gain in visual perception-An EEG study. Neurolmage, 63(1), 223-231. https://doi.org/10.1016/j.neuroimage.2012.06.044

Brown, R. P., Gerbarg, P. L., \& Muench, F. (2013). Breathing Practices for Treatment of Psychiatric and Stress-Related Medical Conditions. Psychiatric Clinics, 36(1), 121-140. https://doi.org/10.1016/j.psc.2013.01.001

Butler, E. A., Wilhelm, F. H., \& Gross, J. J. (2006). Respiratory sinus arrhythmia, emotion, and emotion regulation during social interaction. Psychophysiology, 43(6), 612-622. https://doi.org/10.1111/j.1469-8986.2006.00467.x 
ALLEN ET AL., RESPIRATORY RHYTHMS

Canolty, R. T., \& Knight, R. T. (2010). The functional role of cross-frequency coupling. Trends in Cognitive Sciences, 14(11), 506-515. https://doi.org/10.1016/j.tics.2010.09.001

Chaudhury, S., Sharma, V., Kumar, V., Nag, T. C., \& Wadhwa, S. (2016). Activity-dependent synaptic plasticity modulates the critical phase of brain development. Brain and Development, 38(4), 355-363. https://doi.org/10.1016/j.braindev.2015.10.008

Cheadle, S., Wyart, V., Tsetsos, K., Myers, N., de Gardelle, V., Herce Castañón, S., \& Summerfield, C. (2014). Adaptive Gain Control during Human Perceptual Choice. Neuron, 81(6), 1429-1441. https://doi.org/10.1016/j.neuron.2014.01.020

Chow, B. W., Nuñez, V., Kaplan, L., Granger, A. J., Bistrong, K., Zucker, H. L., Kumar, P., Sabatini, B. L., \& Gu, C. (2020). Caveolae in CNS arterioles mediate neurovascular coupling. Nature, 579(7797), 106-110. https://doi.org/10.1038/s41586-020-2026-1

Clark, A. (2013). Whatever next? Predictive brains, situated agents, and the future of cognitive science. Behavioral and Brain Sciences, 36(3), 181-204. https://doi.org/10.1017/S0140525X12000477

Corlett, P. R., Horga, G., Fletcher, P. C., Alderson-Day, B., Schmack, K., \& Powers, A. R. (2019). Hallucinations and Strong Priors. Trends in Cognitive Sciences, 23(2), 114-127. https://doi.org/10.1016/j.tics.2018.12.001

Craig, A. D. (2002). How do you feel? Interoception: the sense of the physiological condition of the body. Nature Reviews Neuroscience, 3(8), 655-666. https://doi.org/10.1038/nrn894

Del Negro, C. A., Funk, G. D., \& Feldman, J. L. (2018). Breathing matters. Nature Reviews Neuroscience, 19(6), 351-367. https://doi.org/10.1038/s41583-018-0003-6

Eldar, E., Cohen, J. D., \& Niv, Y. (2013). The effects of neural gain on attention and learning. Nature Neuroscience, 16(8), 1146-1153. https://doi.org/10.1038/nn.3428

Eldar, E., Niv, Y., \& Cohen, J. D. (2016). Do You See the Forest or the Tree? Neural Gain and Breadth Versus Focus in Perceptual Processing. Psychological Science, 27(12), 1632-1643. https://doi.org/10.1177/0956797616665578 
ALLEN ET AL., RESPIRATORY RHYTHMS

Evans, K. C., Banzett, R. B., Adams, L., McKay, L., Frackowiak, R. S. J., \& Corfield, D. R. (2002). BOLD fMRI Identifies Limbic, Paralimbic, and Cerebellar Activation During Air Hunger. Journal of Neurophysiology, 88(3), 1500-1511. https://doi.org/10.1152/jn.2002.88.3.1500

Fardo, F., Auksztulewicz, R., Allen, M., Dietz, M. J., Roepstorff, A., \& Friston, K. J. (2017). Expectation violation and attention to pain jointly modulate neural gain in somatosensory cortex. Neurolmage, 153, 109-121.

Faull, O. K., Hayen, A., \& Pattinson, K. T. S. (2017). Breathlessness and the body: Neuroimaging clues for the inferential leap. Cortex, 95, 211-221. https://doi.org/10.1016/j.cortex.2017.07.019 Feldman, H., \& Friston, K. (2010). Attention, Uncertainty, and Free-Energy. Frontiers in Human Neuroscience, 4. https://doi.org/10.3389/fnhum.2010.00215

Ferguson, K. A., \& Cardin, J. A. (2020). Mechanisms underlying gain modulation in the cortex. Nature Reviews Neuroscience, 21(2), 80-92. https://doi.org/10.1038/s41583-019-0253-y

Fox, M. D., \& Raichle, M. E. (2007). Spontaneous fluctuations in brain activity observed with functional magnetic resonance imaging. Nature Reviews Neuroscience, 8(9), 700.

Friston, K. (2013). Life as we know it. Journal of The Royal Society Interface, 10(86), 20130475. https://doi.org/10.1098/rsif.2013.0475

Friston, K. (2018). Does predictive coding have a future? Nature Neuroscience, 21(8), 1019-1021. https://doi.org/10.1038/s41593-018-0200-7

Friston, K. J. (2011). Functional and effective connectivity: A review. Brain Connectivity, 1(1), 13-36. https://doi.org/10.1089/brain.2011.0008

Friston, K. J., Rosch, R., Parr, T., Price, C., \& Bowman, H. (2018). Deep temporal models and active inference. Neuroscience \& Biobehavioral Reviews, 90, 486-501. https://doi.org/10.1016/j.neubiorev.2018.04.004

Friston, K., \& Kiebel, S. (2009). Predictive coding under the free-energy principle. Philosophical Transactions of the Royal Society B: Biological Sciences, 364(1521), 1211-1221. https://doi.org/10.1098/rstb.2008.0300 
ALLEN ET AL., RESPIRATORY RHYTHMS

Galuske, R. A. W., Munk, M. H. J., \& Singer, W. (2019). Relation between gamma oscillations and neuronal plasticity in the visual cortex. Proceedings of the National Academy of Sciences, 116(46), 23317-23325.

Garfinkel, S. N., Manassei, M. F., Hamilton-Fletcher, G., In den Bosch, Y., Critchley, H. D., \& Engels, M. (2016). Interoceptive dimensions across cardiac and respiratory axes. Philosophical Transactions of the Royal Society B: Biological Sciences, 371(1708), 20160014. https://doi.org/10.1098/rstb.2016.0014

Garfinkel, S. N., Seth, A. K., Barrett, A. B., Suzuki, K., \& Critchley, H. D. (2015). Knowing your own heart: Distinguishing interoceptive accuracy from interoceptive awareness. Biological Psychology, 104, 65-74. https://doi.org/10.1016/j.biopsycho.2014.11.004

Giardino, N. D., Curtis, J. L., Abelson, J. L., King, A. P., Pamp, B., Liberzon, I., \& Martinez, F. J. (2010). The impact of panic disorder on interoception and dyspnea reports in chronic obstructive pulmonary disease. Biological Psychology, 84(1), 142-146. https://doi.org/10.1016/j.biopsycho.2010.02.007

Glover, G. H., Li, T.-Q., \& Ress, D. (2000). Image-based method for retrospective correction of physiological motion effects in fMRI: RETROICOR. Magnetic Resonance in Medicine, 44(1), 162-167. https://doi.org/10.1002/1522-2594(200007)44:1<162::AID-MRM23>3.0.CO;2-E

Grossman, D., \& Christensen, L. W. (2007). On combat: The psychology and physiology of deadly conflict in war and in peace. Human Factor Research Group Incorporated.

Grueschow, M., Kleim, B., \& Ruff, C. C. (2020). Role of the locus coeruleus arousal system in cognitive control. Journal of Neuroendocrinology, 32(12), e12890. https://doi.org/10.1111/jne.12890

Grund, M., Al, E., Pabst, M., Dabbagh, A., Stephani, T., Nierhaus, T., Gaebler, M., \& Villringer, A. (2022). Respiration, Heartbeat, and Conscious Tactile Perception. Journal of Neuroscience, 42(4), 643-656. https://doi.org/10.1523/JNEUROSCI.0592-21.2021 


\section{ALLEN ET AL., RESPIRATORY RHYTHMS}

Guggenmos, M., Wilbertz, G., Hebart, M. N., \& Sterzer, P. (2016). Mesolimbic confidence signals guide perceptual learning in the absence of external feedback. ELife, 5, e13388. https://doi.org/10.7554/eLife.13388

Harrison, O. K., Garfinkel, S. N., Marlow, L., Finnegan, S., Marino, S., Nanz, L., Allen, M., Finnemann, J., Keur-Huizinga, L., Harrison, S. J., Stephan, K. E., Pattinson, K. T. S., \& Fleming, S. M. (2021). The Filter Detection Task for measurement of breathing-related interoception and metacognition. BioRxiv, 2020.06.29.176941. https://doi.org/10.1101/2020.06.29.176941

Harrison, O. K., Köchli, L., Marino, S., Luechinger, R., Hennel, F., Brand, K., Hess, A. J., Frässle, S., Iglesias, S., Vinckier, F., Petzschner, F. H., Harrison, S. J., \& Stephan, K. E. (2021). Interoception of breathing and its relationship with anxiety. Neuron, 109(24), 4080-4093.e8. https://doi.org/10.1016/j.neuron.2021.09.045

Harrison, O. K., Nanz, L., Marino, S., Lüchinger, R., Hennel, F., Hess, A. J., Frässle, S., Iglesias, S., Vinckier, F., Petzschner, F., Harrison, S. J., \& Stephan, K. E. (2021). Interoception of breathing and its relationship with anxiety. BioRxiv, 2021.03.24.436881. https://doi.org/10.1101/2021.03.24.436881

Hauser, T. U., Fiore, V. G., Moutoussis, M., \& Dolan, R. J. (2016). Computational Psychiatry of ADHD: Neural Gain Impairments across Marrian Levels of Analysis. Trends in Neurosciences, 39(2), 63-73. https://doi.org/10.1016/j.tins.2015.12.009

Heck, D. H., Kozma, R., \& Kay, L. M. (2019). The rhythm of memory: How breathing shapes memory function. Journal of Neurophysiology, 122(2), 563-571. https://doi.org/10.1152/jn.00200.2019

Heck, D. H., McAfee, S. S., Liu, Y., Babajani-Feremi, A., Rezaie, R., Freeman, W. J., Wheless, J. W., Papanicolaou, A. C., Ruszinkó, M., Sokolov, Y., \& Kozma, R. (2017). Breathing as a Fundamental Rhythm of Brain Function. Frontiers in Neural Circuits, 10. https://doi.org/10.3389/fncir.2016.00115 
ALLEN ET AL., RESPIRATORY RHYTHMS

Herrero, J. L., Khuvis, S., Yeagle, E., Cerf, M., \& Mehta, A. D. (2017). Breathing above the brainstem: Volitional control and attentional modulation in humans. Journal of Neurophysiology.

Hesp, C., Smith, R., Parr, T., Allen, M., Friston, K. J., \& Ramstead, M. J. D. (2021). Deeply Felt Affect: The Emergence of Valence in Deep Active Inference. Neural Computation, 33(2), 398-446. https://doi.org/10.1162/neco_a_01341

Hohwy, J. (2013). The Predictive Mind. Oxford University Press.

Homma, I., \& Masaoka, Y. (2008). Breathing rhythms and emotions. Experimental Physiology, 93(9), 1011-1021. https://doi.org/10.1113/expphysiol.2008.042424

Hsia, C. C. W., Schmitz, A., Lambertz, M., Perry, S. F., \& Maina, J. N. (2013). Evolution of Air Breathing: Oxygen Homeostasis and the Transitions from Water to Land and Sky. Comprehensive Physiology, 3(2), 849-915. https://doi.org/10.1002/cphy.c120003 Huijbers, W., Pennartz, C. M. A., Beldzik, E., Domagalik, A., Vinck, M., Hofman, W. F., Cabeza, R., \& Daselaar, S. M. (2014). Respiration phase-locks to fast stimulus presentations: Implications for the interpretation of posterior midline "deactivations." Human Brain Mapping, 35(9), 4932-4943. https://doi.org/10.1002/hbm.22523

Insausti, R., Marcos, P., Arroyo-Jiménez, M. M., Blaizot, X., \& Martínez-Marcos, A. (2002). Comparative aspects of the olfactory portion of the entorhinal cortex and its projection to the hippocampus in rodents, nonhuman primates, and the human brain. Brain Research Bulletin, 57(3), 557-560. https://doi.org/10.1016/S0361-9230(01)00684-0

Ito, J., Roy, S., Liu, Y., Cao, Y., Fletcher, M., Lu, L., Boughter, J. D., Grün, S., \& Heck, D. H. (2014). Whisker barrel cortex delta oscillations and gamma power in the awake mouse are linked to respiration. Nature Communications, 5(1), 3572. https://doi.org/10.1038/ncomms4572

Jella, S. A., \& Shannahoff-khalsa, D. S. (1993). The Effects of Unilateral Forced Nostril Breathing on Cognitive Performance. International Journal of Neuroscience, 73(1-2), 61-68. https://doi.org/10.3109/00207459308987211 


\section{ALLEN ET AL., RESPIRATORY RHYTHMS}

Johannknecht, M., \& Kayser, C. (2022). The influence of the respiratory cycle on reaction times in sensory-cognitive paradigms. Scientific Reports, 12(1), 2586. https://doi.org/10.1038/s41598-022-06364-8

Kanai, R., Komura, Y., Shipp, S., \& Friston, K. (2015). Cerebral hierarchies: Predictive processing, precision and the pulvinar. Philosophical Transactions of the Royal Society B: Biological Sciences, 370(1668), 20140169. https://doi.org/10.1098/rstb.2014.0169

Karbowski, J. (2009). Thermodynamic constraints on neural dimensions, firing rates, brain temperature and size. Journal of Computational Neuroscience, 27(3), 415-436. https://doi.org/10.1007/s10827-009-0153-7

Kay, L. M. (2014). Chapter 9-Circuit Oscillations in Odor Perception and Memory. In E. Barkai \& D. A. Wilson (Eds.), Progress in Brain Research (Vol. 208, pp. 223-251). Elsevier. https://doi.org/10.1016/B978-0-444-63350-7.00009-7

Kay, L. M., Beshel, J., Brea, J., Martin, C., Rojas-Líbano, D., \& Kopell, N. (2009). Olfactory oscillations: The what, how and what for. Trends in Neurosciences, 32(4), 207-214. https://doi.org/10.1016/j.tins.2008.11.008

Kellner, R., Samet, J., \& Pathak, D. (1992). Dyspnea, anxiety, and depression in chronic respiratory impairment. General Hospital Psychiatry, 14(1), 20-28. https://doi.org/10.1016/01638343(92)90022-3

Kepecs, A., Uchida, N., \& Mainen, Z. F. (2006). The Sniff as a Unit of Olfactory Processing. Chemical Senses, 31(2), 167-179. https://doi.org/10.1093/chemse/bjj016

Khalsa, S. S., Adolphs, R., Cameron, O. G., Critchley, H. D., Davenport, P. W., Feinstein, J. S., Feusner, J. D., Garfinkel, S. N., Lane, R. D., Mehling, W. E., Meuret, A. E., Nemeroff, C. B., Oppenheimer, S., Petzschner, F. H., Pollatos, O., Rhudy, J. L., Schramm, L. P., Simmons, W. K., Stein, M. B., ... Zucker, N. (2018). Interoception and Mental Health: A Roadmap. Biological Psychiatry: Cognitive Neuroscience and Neuroimaging, 3(6), 501-513. https://doi.org/10.1016/j.bpsc.2017.12.004 
ALLEN ET AL., RESPIRATORY RHYTHMS

Kluger, D. S., Balestrieri, E., Busch, N. A., \& Gross, J. (2021a). Respiration aligns perception with neural excitability. BioRxiv, 2021.03.25.436938. https://doi.org/10.1101/2021.03.25.436938

Kluger, D. S., Balestrieri, E., Busch, N. A., \& Gross, J. (2021b). Respiration aligns perception with neural excitability. ELife, 10, e70907. https://doi.org/10.7554/eLife.70907

Kluger, D. S., \& Gross, J. (2020a). Respiration modulates oscillatory neural network activity at rest. BioRxiv, 2020.04.23.057216. https://doi.org/10.1101/2020.04.23.057216

Kluger, D. S., \& Gross, J. (2020b). Depth and phase of respiration modulate cortico-muscular communication. Neurolmage, 222, 117272. https://doi.org/10.1016/j.neuroimage.2020.117272

Konttinen, N., \& Lyytinen, H. (1992). Physiology of preparation: Brain slow waves, heart rate, and respiration preceding triggering in rifle shooting. International Journal of Sport Psychology, 23(2), 110-127.

Körding, K. P., \& Wolpert, D. M. (2004). Bayesian integration in sensorimotor learning. Nature, 427(6971), 244-247. https://doi.org/10.1038/nature02169

Lega, B., Burke, J., Jacobs, J., \& Kahana, M. J. (2016). Slow-Theta-to-Gamma Phase-Amplitude Coupling in Human Hippocampus Supports the Formation of New Episodic Memories. Cerebral Cortex, 26(1), 268-278. https://doi.org/10.1093/cercor/bhu232

Legrand, N., Nikolova, N., Correa, C., Brændholt, M., Stuckert, A., Kildahl, N., Vejlø, M., Fardo, F., \& Allen, M. (2021). The heart rate discrimination task: A psychophysical method to estimate the accuracy and precision of interoceptive beliefs. Biological Psychology, 108239. https://doi.org/10.1016/j.biopsycho.2021.108239

Lisman, J. E., \& Jensen, O. (2013). The Theta-Gamma Neural Code. Neuron, 77(6), 1002-1016. https://doi.org/10.1016/j.neuron.2013.03.007

Liu, Y., McAfee, S. S., \& Heck, D. H. (2017). Hippocampal sharp-wave ripples in awake mice are entrained by respiration. Scientific Reports, 7(1), 1-9. https://doi.org/10.1038/s41598-01709511-8 
ALLEN ET AL., RESPIRATORY RHYTHMS

Manning, H. L., \& Schwartzstein, R. M. (1995). Pathophysiology of Dyspnea. New England Journal of Medicine, 333(23), 1547-1553. https://doi.org/10.1056/NEJM199512073332307

Mather, M., Clewett, D., Sakaki, M., \& Harley, C. W. (2016). Norepinephrine ignites local hotspots of neuronal excitation: How arousal amplifies selectivity in perception and memory. Behavioral and Brain Sciences, 39. https://doi.org/10.1017/\$0140525X15000667

McKay, L. C., Evans, K. C., Frackowiak, R. S. J., \& Corfield, D. R. (2003). Neural correlates of voluntary breathing in humans. Journal of Applied Physiology, 95(3), 1170-1178. https://doi.org/10.1152/japplphysiol.00641.2002

Mizuhara, K., \& Nittono, H. (2022). Visual discrimination accuracy does not differ between nasal inhalation and exhalation when stimuli are voluntarily aligned to breathing phase. International Journal of Psychophysiology, 173, 1-8. https://doi.org/10.1016/j.ijpsycho.2021.12.009

Moberly, A. H., Schreck, M., Bhattarai, J. P., Zweifel, L. S., Luo, W., \& Ma, M. (2018). Olfactory inputs modulate respiration-related rhythmic activity in the prefrontal cortex and freezing behavior. Nature Communications, 9(1), 1528. https://doi.org/10.1038/s41467-018-03988-1

Molle, L., \& Coste, A. (2022). The respiratory modulation of interoception. Journal of Neurophysiology, 127(4), 896-899. https://doi.org/10.1152/jn.00027.2022

Monti, A., Porciello, G., Tieri, G., \& Aglioti, S. M. (2019). The "embreathment" illusion highlights the role of breathing in corporeal awareness. Journal of Neurophysiology, 123(1), 420-427. https://doi.org/10.1152/jn.00617.2019

Moran, R. J., Campo, P., Symmonds, M., Stephan, K. E., Dolan, R. J., \& Friston, K. J. (2013a). Free Energy, Precision and Learning: The Role of Cholinergic Neuromodulation. Journal of Neuroscience, 33(19), 8227-8236. https://doi.org/10.1523/JNEUROSCI.4255-12.2013

Moran, R. J., Campo, P., Symmonds, M., Stephan, K. E., Dolan, R. J., \& Friston, K. J. (2013b). Free Energy, Precision and Learning: The Role of Cholinergic Neuromodulation. Journal of Neuroscience, 33(19), 8227-8236. https://doi.org/10.1523/JNEUROSCI.4255-12.2013 
ALLEN ET AL., RESPIRATORY RHYTHMS

Naidu, S. B., Shah, A. J., Saigal, A., Smith, C., Brill, S. E., Goldring, J., Hurst, J. R., Jarvis, H., Lipman, M., \& Mandal, S. (2021). The high mental health burden of "Long COVID" and its association with on-going physical and respiratory symptoms in all adults discharged from hospital. European Respiratory Journal. https://doi.org/10.1183/13993003.04364-2020

Nakamura, N. H., Fukunaga, M., \& Oku, Y. (2018). Respiratory modulation of cognitive performance during the retrieval process. PLOS ONE, 13(9), e0204021.

https://doi.org/10.1371/journal.pone.0204021

Nigri, A., Ferraro, S., D’Incerti, L., Critchley, H. D., Bruzzone, M. G., \& Minati, L. (2013). Connectivity of the amygdala, piriform, and orbitofrontal cortex during olfactory stimulation: A functional MRI study. NeuroReport, 24(4), 171-175. https://doi.org/10.1097/WNR.0b013e32835d5d2b

Nikolova, N., Harrison, O., Toohey, S., Brændholt, M., Legrand, N., Correa, C., Vejlø, M., Jensen, M. S., Fardo, F., \& Allen, M. (2022). The respiratory resistance sensitivity task: An automated method for quantifying respiratory interoception and metacognition. Biological Psychology, 170, 108325. https://doi.org/10.1016/j.biopsycho.2022.108325

Nikolova, N., Waade, P. T., Friston, K. J., \& Allen, M. (2021). What Might Interoceptive Inference Reveal about Consciousness? Review of Philosophy and Psychology. https://doi.org/10.1007/s13164-021-00580-3

Orepic, P., Park, H.-D., Rognini, G., Faivre, N., \& Blanke, O. (2022). Breathing affects self-other voice discrimination in a bodily state associated with somatic passivity. Psychophysiology, n/a(n/a), e14016. https://doi.org/10.1111/psyp.14016

Osborne, J. M., Bernabeu, M. O., Bruna, M., Calderhead, B., Cooper, J., Dalchau, N., Dunn, S.-J., Fletcher, A. G., Freeman, R., Groen, D., Knapp, B., Mclnerny, G. J., Mirams, G. R., Pitt-Francis, J., Sengupta, B., Wright, D. W., Yates, C. A., Gavaghan, D. J., Emmott, S., \& Deane, C. (2014). Ten Simple Rules for Effective Computational Research. PLOS Computational Biology, 10(3), e1003506. https://doi.org/10.1371/journal.pcbi.1003506 


\section{ALLEN ET AL., RESPIRATORY RHYTHMS}

Owens, A. P., Allen, M., Ondobaka, S., \& Friston, K. J. (2018). Interoceptive inference: From computational neuroscience to clinic. Neuroscience \& Biobehavioral Reviews, 90, 174-183. https://doi.org/10.1016/j.neubiorev.2018.04.017

Palminteri, S., Wyart, V., \& Koechlin, E. (2017). The Importance of Falsification in Computational Cognitive Modeling. Trends in Cognitive Sciences, 21(6), 425-433. https://doi.org/10.1016/j.tics.2017.03.011

Park, H.-D., Barnoud, C., Trang, H., Kannape, O. A., Schaller, K., \& Blanke, O. (2020). Breathing is coupled with voluntary action and the cortical readiness potential. Nature Communications, 11(1), 1-8. https://doi.org/10.1038/s41467-019-13967-9

Parr, T., \& Friston, K. J. (2017). Uncertainty, epistemics and active inference. Journal of The Royal Society Interface, 14(136), 20170376. https://doi.org/10.1098/rsif.2017.0376

Perl, O., Ravia, A., Rubinson, M., Eisen, A., Soroka, T., Mor, N., Secundo, L., \& Sobel, N. (2019). Human non-olfactory cognition phase-locked with inhalation. Nature Human Behaviour, 3(5), 501-512. https://doi.org/10.1038/s41562-019-0556-z

Petzschner, F. H., Garfinkel, S. N., Paulus, M. P., Koch, C., \& Khalsa, S. S. (2021). Computational Models of Interoception and Body Regulation. Trends in Neurosciences, 44(1), 63-76. https://doi.org/10.1016/j.tins.2020.09.012

Pinotsis, D. A., Loonis, R., Bastos, A. M., Miller, E. K., \& Friston, K. J. (2019). Bayesian modelling of induced responses and neuronal rhythms. Brain Topography, 32(4), 569-582.

Potter, H., \& Nauta, W. J. H. (1979). A note on the problem of olfactory associations of the orbitofrontal cortex in the monkey. Neuroscience, 4(3), 361-367. https://doi.org/10.1016/0306-4522(79)90099-X

Pulcu, E., \& Browning, M. (2019). The Misestimation of Uncertainty in Affective Disorders. Trends in Cognitive Sciences, 23(10), 865-875. https://doi.org/10.1016/j.tics.2019.07.007 
ALLEN ET AL., RESPIRATORY RHYTHMS

Rao, R. P. N., \& Ballard, D. H. (1999). Predictive coding in the visual cortex: A functional interpretation of some extra-classical receptive-field effects. Nature Neuroscience, 2(1), 7987. https://doi.org/10.1038/4580

Rebola, N., Srikumar, B. N., \& Mulle, C. (2010). Activity-dependent synaptic plasticity of NMDA receptors. The Journal of Physiology, 588(1), 93-99. https://doi.org/10.1113/jphysiol.2009.179382

Servan-Schreiber, D., Printz, H., \& Cohen, J. D. (1990). A network model of catecholamine effects: Gain, signal-to-noise ratio, and behavior. Science (New York, N.Y.), 249(4971), 892-895. https://doi.org/10.1126/science.2392679

Seth, A. K. (2013). Interoceptive inference, emotion, and the embodied self. Trends in Cognitive Sciences, 17(11), 565-573. https://doi.org/10.1016/j.tics.2013.09.007

Seth, A. K., \& Tsakiris, M. (2018). Being a Beast Machine: The Somatic Basis of Selfhood. Trends in Cognitive Sciences, 22(11), 969-981. https://doi.org/10.1016/j.tics.2018.08.008

Shine, J. M., Aburn, M. J., Breakspear, M., \& Poldrack, R. A. (2018). The modulation of neural gain facilitates a transition between functional segregation and integration in the brain. ELife, 7 , e31130. https://doi.org/10.7554/eLife.31130

Shine, J. M., Bell, P. T., Matar, E., Poldrack, R. A., Lewis, S. J. G., Halliday, G. M., \& O'Callaghan, C. (2019). Dopamine depletion alters macroscopic network dynamics in Parkinson's disease. Brain, 142(4), 1024-1034. https://doi.org/10.1093/brain/awz034

Shine, J. M., Bissett, P. G., Bell, P. T., Koyejo, O., Balsters, J. H., Gorgolewski, K. J., Moodie, C. A., \& Poldrack, R. A. (2016). The Dynamics of Functional Brain Networks: Integrated Network States during Cognitive Task Performance. Neuron, 92(2), 544-554. https://doi.org/10.1016/j.neuron.2016.09.018

Smith, R., Mayeli, A., Taylor, S., Al Zoubi, O., Naegele, J., \& Khalsa, S. S. (2021). Gut inference: A computational modelling approach. Biological Psychology, 164, 108152. https://doi.org/10.1016/j.biopsycho.2021.108152 


\section{ALLEN ET AL., RESPIRATORY RHYTHMS}

Smith, R., Thayer, J. F., Khalsa, S. S., \& Lane, R. D. (2017). The hierarchical basis of neurovisceral integration. Neuroscience \& Biobehavioral Reviews, 75, 274-296. https://doi.org/10.1016/j.neubiorev.2017.02.003

Sobel, N., Prabhakaran, V., Desmond, J. E., Glover, G. H., Goode, R. L., Sullivan, E. V., \& Gabrieli, J. D. E. (1998). Sniffing and smelling: Separate subsystems in the human olfactory cortex. Nature, 392(6673), 282-286. https://doi.org/10.1038/32654

Stančák, A., \& Kuna, M. (1994). EEG changes during forced alternate nostril breathing. International Journal of Psychophysiology, 18(1), 75-79. https://doi.org/10.1016/0167-8760(84)90017-5

Stephan, K. E., Manjaly, Z. M., Mathys, C. D., Weber, L. A. E., Paliwal, S., Gard, T., Tittgemeyer, M., Fleming, S. M., Haker, H., Seth, A. K., \& Petzschner, F. H. (2016). Allostatic Self-efficacy: A Metacognitive Theory of Dyshomeostasis-Induced Fatigue and Depression. Frontiers in Human Neuroscience, 10. https://doi.org/10.3389/fnhum.2016.00550

Sudre, C. H., Murray, B., Varsavsky, T., Graham, M. S., Penfold, R. S., Bowyer, R. C., Pujol, J. C., Klaser, K., Antonelli, M., Canas, L. S., Molteni, E., Modat, M., Jorge Cardoso, M., May, A., Ganesh, S., Davies, R., Nguyen, L. H., Drew, D. A., Astley, C. M., ... Steves, C. J. (2021). Attributes and predictors of long COVID. Nature Medicine, 27(4), 626-631. https://doi.org/10.1038/s41591021-01292-y

Taquet, M., Luciano, S., Geddes, J. R., \& Harrison, P. J. (2021). Bidirectional associations between COVID-19 and psychiatric disorder: Retrospective cohort studies of 62354 COVID-19 cases in the USA. The Lancet Psychiatry, 8(2), 130-140. https://doi.org/10.1016/S22150366(20)30462-4

Tort, A. B. L., Brankačk, J., \& Draguhn, A. (2018). Respiration-Entrained Brain Rhythms Are Global but Often Overlooked. Trends in Neurosciences, 41(4), 186-197. https://doi.org/10.1016/j.tins.2018.01.007

Tort, A. B. L., Komorowski, R. W., Manns, J. R., Kopell, N. J., \& Eichenbaum, H. (2009). Theta-gamma coupling increases during the learning of item-context associations. Proceedings of the 


\section{ALLEN ET AL., RESPIRATORY RHYTHMS}

National Academy of Sciences of the United States of America, 106(49), 20942-20947. https://doi.org/10.1073/pnas.0911331106

Tort, A. B. L., Kramer, M. A., Thorn, C., Gibson, D. J., Kubota, Y., Graybiel, A. M., \& Kopell, N. J. (2008). Dynamic cross-frequency couplings of local field potential oscillations in rat striatum and hippocampus during performance of a T-maze task. Proceedings of the National Academy of Sciences, 105(51), 20517-20522. https://doi.org/10.1073/pnas.0810524105

Tweeddale, P. M., Rowbottom, I., \& McHardy, G. J. R. (1994). Breathing retraining: Effect on anxiety and depression scores in behavioural breathlessness. Journal of Psychosomatic Research, 38(1), 11-21. https://doi.org/10.1016/0022-3999(94)90004-3

Varga, S., \& Heck, D. H. (2017). Rhythms of the body, rhythms of the brain: Respiration, neural oscillations, and embodied cognition. Consciousness and Cognition, 56, 77-90. https://doi.org/10.1016/j.concog.2017.09.008

Vinck, M., Batista-Brito, R., Knoblich, U., \& Cardin, J. A. (2015). Arousal and Locomotion Make Distinct Contributions to Cortical Activity Patterns and Visual Encoding. Neuron, 86(3), 740754. https://doi.org/10.1016/j.neuron.2015.03.028

von Leupoldt, A., Chan, P.-Y. S., Bradley, M. M., Lang, P. J., \& Davenport, P. W. (2011). The impact of anxiety on the neural processing of respiratory sensations. Neurolmage, 55(1), 247-252. https://doi.org/10.1016/j.neuroimage.2010.11.050

Warren, C. M., Eldar, E., van den Brink, R. L., Tona, K.-D., van der Wee, N. J., Giltay, E. J., Van Noorden, M. S., Bosch, J. A., Wilson, R. C., \& Cohen, J. D. (2016). Catecholamine-mediated increases in gain enhance the precision of cortical representations. Journal of Neuroscience, 36(21), 5699-5708.

Whyte, C. J., \& Smith, R. (2021). The predictive global neuronal workspace: A formal active inference model of visual consciousness. Progress in Neurobiology, 199, 101918. https://doi.org/10.1016/j.pneurobio.2020.101918 


\section{ALLEN ET AL., RESPIRATORY RHYTHMS}

Wilson, R. C., \& Collins, A. G. (2019). Ten simple rules for the computational modeling of behavioral data. ELife, 8, e49547. https://doi.org/10.7554/eLife.49547

Wolpert, D. M. (1997). Computational approaches to motor control. Trends in Cognitive Sciences, 1(6), 209-216. https://doi.org/10.1016/S1364-6613(97)01070-X

Yackle, K., Schwarz, L. A., Kam, K., Sorokin, J. M., Huguenard, J. R., Feldman, J. L., Luo, L., \& Krasnow, M. A. (2017). Breathing control center neurons that promote arousal in mice. Science, 355(6332), 1411-1415. https://doi.org/10.1126/science.aai7984

Zaccaro, A., Perrucci, M. G., Parrotta, E., Costantini, M., \& Ferri, F. (2022). Brain-heart interactions are optimized across the respiratory cycle via interoceptive attention (p. 2022.04.02.486808). bioRxiv. https://doi.org/10.1101/2022.04.02.486808

Zelano, C., Jiang, H., Zhou, G., Arora, N., Schuele, S., Rosenow, J., \& Gottfried, J. A. (2016). Nasal Respiration Entrains Human Limbic Oscillations and Modulates Cognitive Function. Journal of Neuroscience, 36(49), 12448-12467. https://doi.org/10.1523/JNEUROSCI.2586-16.2016

Zerbi, V., Floriou-Servou, A., Markicevic, M., Vermeiren, Y., Sturman, O., Privitera, M., von Ziegler, L., Ferrari, K. D., Weber, B., \& De Deyn, P. P. (2019). Rapid reconfiguration of the functional connectome after chemogenetic locus coeruleus activation. Neuron, 103(4), 702-718. 
Figures and Figure Legends

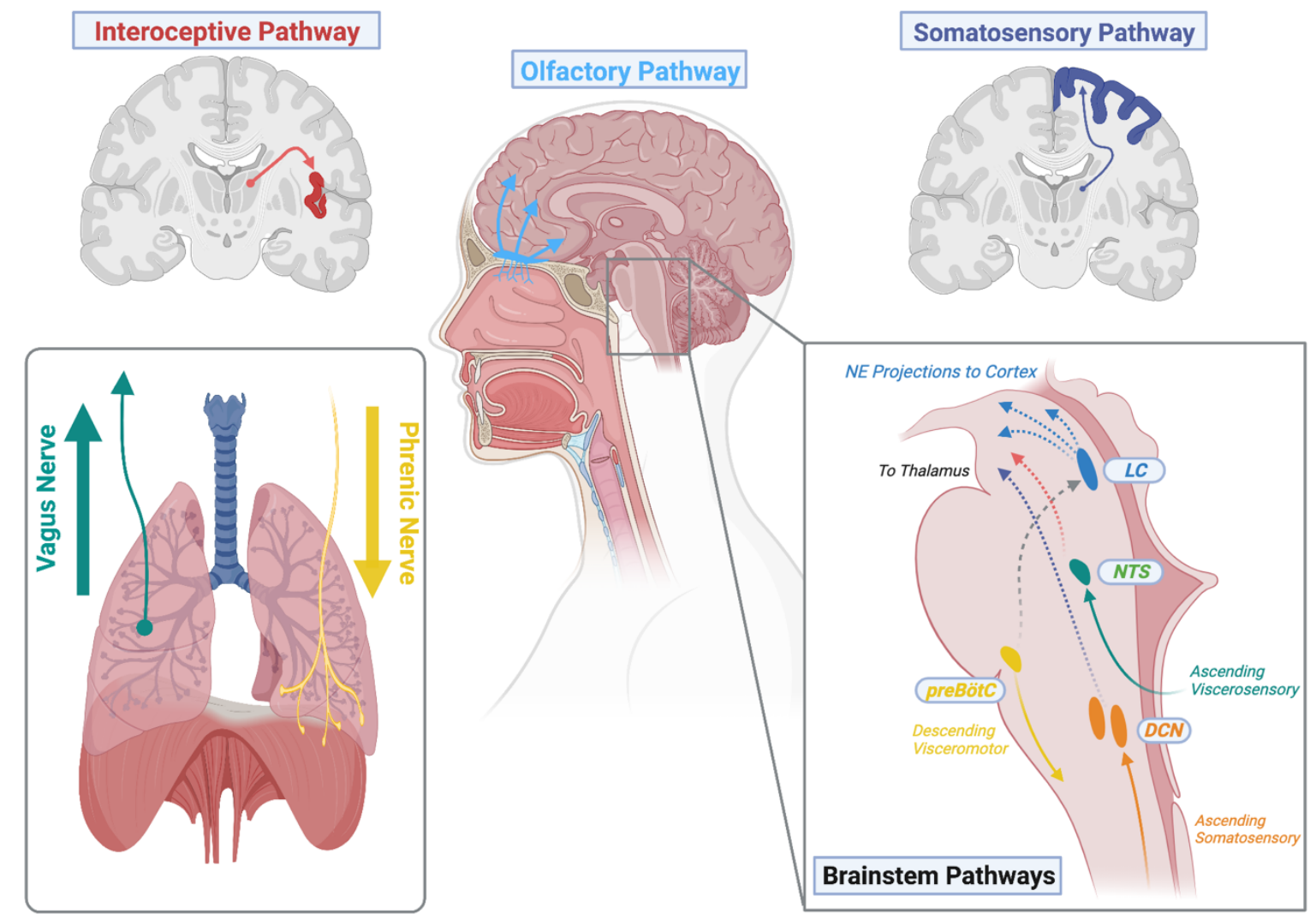

Figure 1: Major Neuroanatomical Pathways Determining Respiratory-Brain Coupling.

Respiratory rhythms can constrain brain function by at least three interacting neuroanatomical pathways. At each respiratory cycle, descending motor signals originating in the pre-Bötzinger complex (preBötC) are carried to the lungs and diaphragm via the phrenic nerve, eliciting muscular contraction and relaxation. Each breath then drives rhythmic 


\section{ALLEN ET AL., RESPIRATORY RHYTHMS}

olfactory, somatosensory, and interoceptive signals which are communicated to the brain via the olfactory bulb, somatosensory, and vagal nerves respectively. The mechanical ventilation of the lungs induces regular somatosensory rhythms in the chest wall, head, and bodily posture, which are relayed by the dorsal column nuclei (DCN) to engage beta oscillations in the primary somatosensory cortex and cerebellum, synchronizing sensorimotor rhythms with the breath. In the brainstem, a complex predictive control loop between the various respiratory nuclei integrates ascending viscerosensory and descending visceromotor signals to maintain respiratory homeostasis. This loop also directly engages the locus coeruleus (LC), linking respiratory cycles to global noradrenergic (NE) gain control (shown in blue). The rhythmic fluctuation of homeostatic variables such as air hunger and respiratory effort is relayed to the nucleus tractus solitarius (NTS) of the brainstem by the ascending vagus (pathway in green), before being transmitted to higher interoceptive regions such as the insular cortex (illustrated in red). 


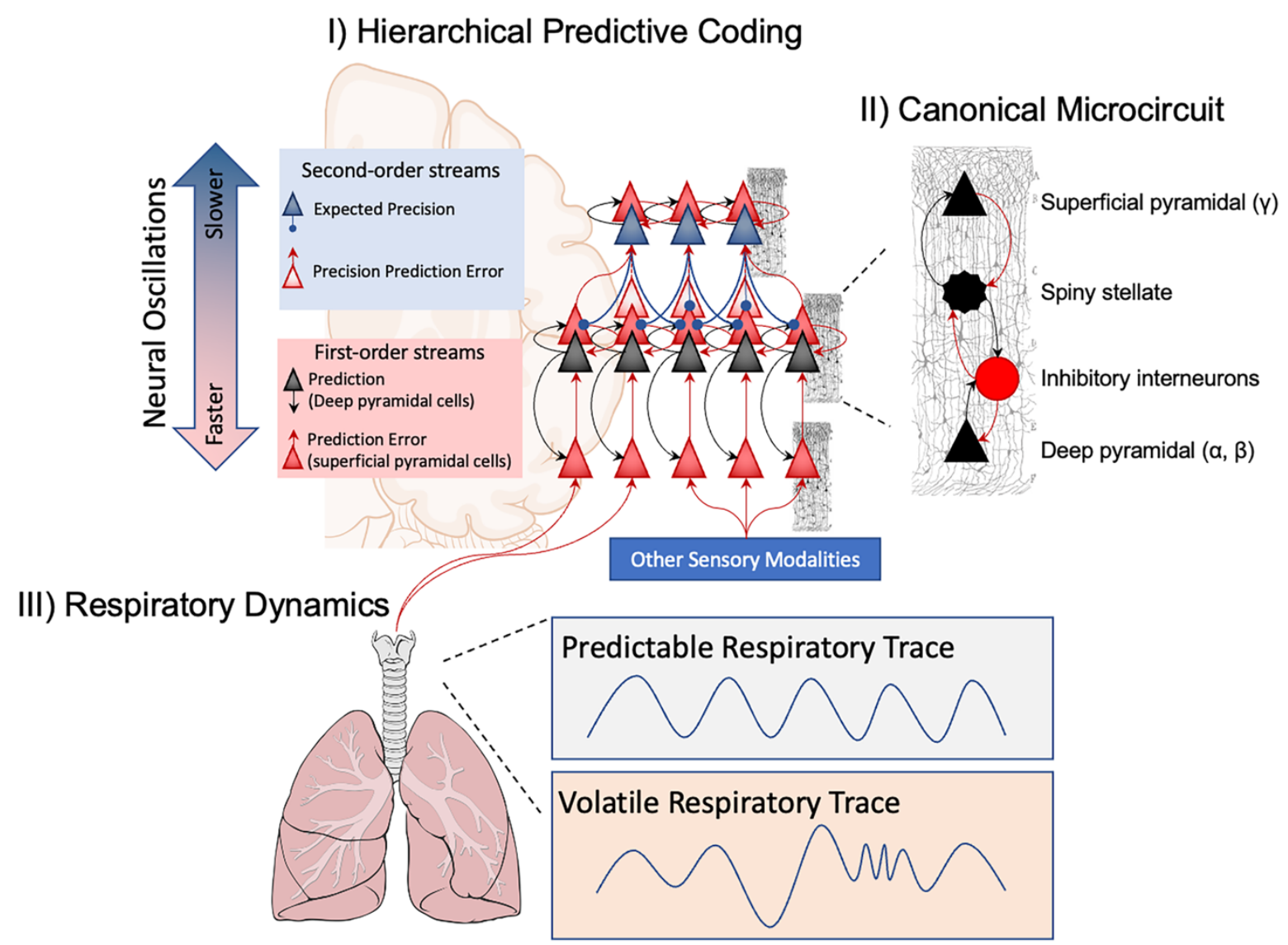

Figure 2 Hierarchical Predictive Coding and Respiratory-Brain Coupling. I) Ascending prediction errors encode interoceptive (e.g., respiratory) and exteroceptive (e.g., visual) sensory inputs in an ascending stream of ever-more abstract representations. First order predictions encode low-level deviations in respiratory parameters such as blood oxygenation or moment-to-moment respiratory depth. As one moves along the cortical hierarchy, from first to second-order prediction-streams, predictions become more abstract, encoding the expected volatility or predictability of respiration in a given context, rather than the immediate stream of sensory inputs. II) This scheme of hierarchical message passing is accomplished by a recurrent motif of canonical microcircuits, in which predictions, prediction errors, and their precision are encoded by specific neural populations that exhibit unique temporal synchrony profiles such that more superficial cortical layers encode 


\section{ALLEN ET AL., RESPIRATORY RHYTHMS}

prediction errors via fast spiking oscillations (e.g., gamma, $\gamma$ ) and deeper levels encode predictions via slower rhythms (e.g., alpha, $\alpha$, beta, $\beta$ ). III) Within this scheme, respiratory dynamics are likely to exert several unique influences on neuronal and computational function. For example, the statistical properties of increasingly volatile respiration (e.g., mean, and variance) will elicit viscero-sensory prediction errors and/or precision-prediction errors that will not only alter respiratory phenomenology and control, but also potentially other exteroceptive or affective representations linked through lateral connectivity. Additionally, by altering structural and physiological properties of brain tissue, sudden or chronic changes in respiration can alter the excitability of different cortical layers, causing heterogeneous but global changes in the precision of descending predictions or ascending prediction errors, respectively. 


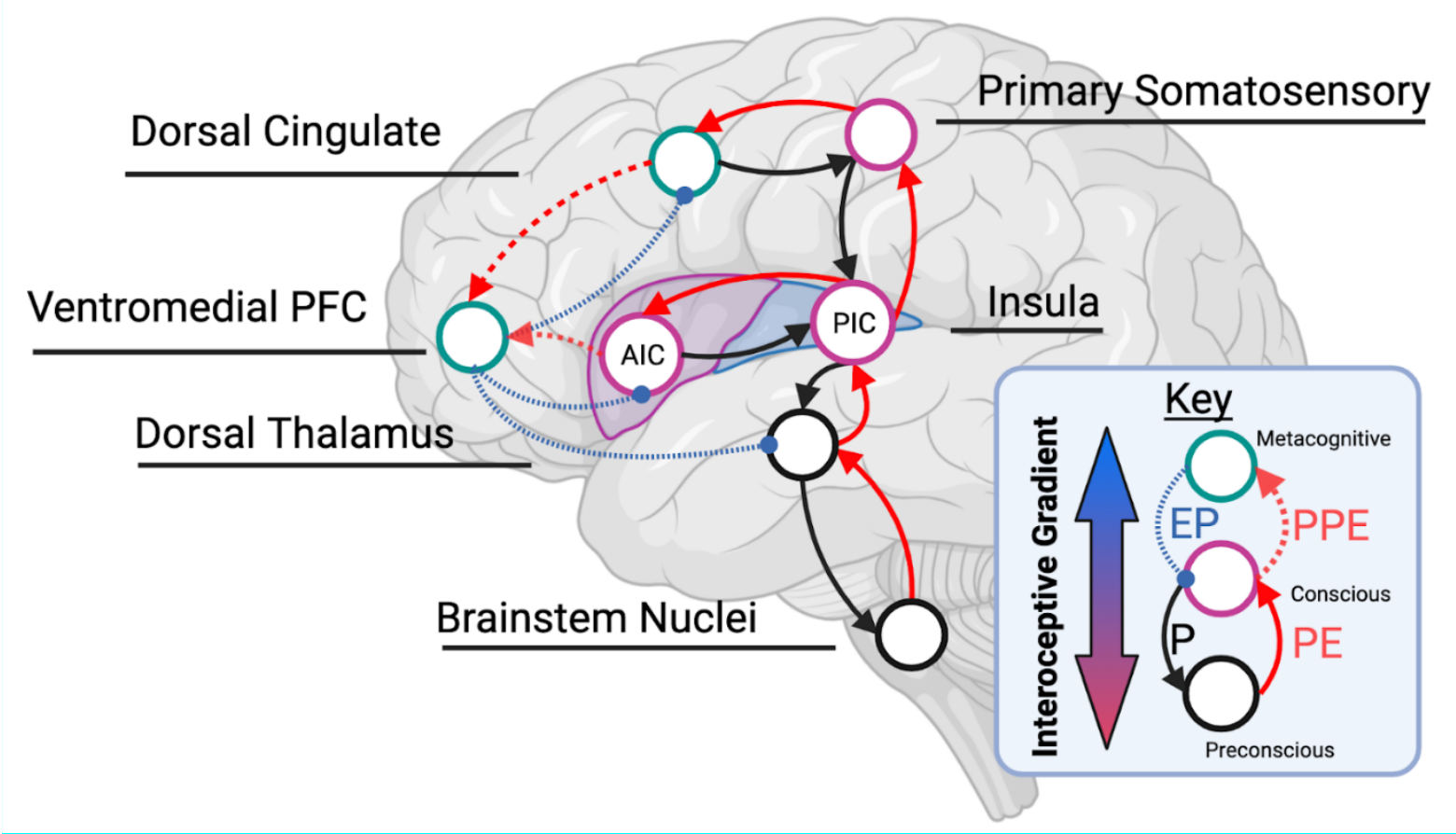

Figure 3: Mapping Respiratory Rhythms onto a Gradient of Interocpetive Inference.

Here we depict a putative cortical-subcortical hierarchy to illustrate how the three anatomical pathways dominating respiratory-modulated brain oscillations may influence interoceptive inference and learning. On our model, a low-level respiratory comparator involving thalamic and brainstem nuclei monitors and controls respiratory physiology within homeostatic setpoints. This low-level loop gives off prediction errors which are processed by associative somatomotor and interoceptive loops in the somatosensory, cingulate, and insular cortices. Here, multimodal interoceptive predictions integrate exteroceptive, somatomotor, and interoceptive prediction errors from primary sensory regions. Finally, metacognitive awareness, control, and learning are instantiated by the ventromedial prefrontal cortex through the estimation of precision prediction errors; by controlling neuromodulators, the loop is completed such that high level interoceptive beliefs can constrain the excitability of the lower and mid-level regions. By comparison to Figure 1, this model predicts specific pathways of respiratory interaction; intranasal effects would be mapped onto metacognitive 
ALLEN ET AL., RESPIRATORY RHYTHMS

regions, somatomotor onto the mid-level, and arousal-mediated effects more globally constraining neural gain throughout the hierarchy. 